\title{
Distorted Mix Method for Constructing Copulas with Tail Dependence
}

\author{
Lujun $\mathrm{Li}^{*}$
}

\author{
K. C. Yuen ${ }^{\dagger}$
}

April 2014

\author{
Jingping Yang
}

\begin{abstract}
This paper introduces a method for constructing copula functions by combining the ideas of distortion and convex sum, named Distorted Mix Method. The method mixes different copulas with distorted margins to construct new copula functions, and it enables us to model the dependence structure of risks by handling central and tail parts separately. By applying the method we can modify the tail dependence of a given copula to any desired level measured by tail dependence function and tail dependence coefficients of marginal distributions. As an application, a tight bound for asymptotic Value-at-Risk of order statistics is obtained by using the method. An empirical study shows that copulas constructed by this method fit the empirical data of SPX 500 Index and FTSE 100 Index very well in both central and tail parts.
\end{abstract}

Keywords: copula; Distorted Mix Method; distortion function; tail dependence coefficient; tail dependence function.

\section{Introduction}

Copula method can be applied for describing the full dependence structure of random vectors. Essentially, a copula function is a joint distribution with all uniform $[0,1]$ margins. It is known that the copula function provides a complete description of dependence structure among random variables by uniting marginal distributions via Sklar Theorem. Let $F\left(x_{1}, \ldots, x_{d}\right)$ be a $d$-dimensional joint distribution with marginal distributions $F_{1}\left(x_{1}\right), \ldots, F_{d}\left(x_{d}\right)$. Sklar Theorem states that there exists a copula function $C$ satisfying

$$
F\left(x_{1}, \ldots, x_{d}\right)=C\left(F_{1}\left(x_{1}\right), \ldots, F_{d}\left(x_{d}\right)\right) .
$$

${ }^{*}$ Department of Financial Mathematics, Peking University, Beijing 100871, China. Email address: lujun.li@pku.edu.cn

${ }^{\dagger}$ Department of Statistics and Actuarial Science, The University of Hong Kong, Pokfulam Road, Hong Kong. Email address: kcyuen@hku.hk

${ }^{\ddagger}$ LMEQF, Department of Financial Mathematics, Peking University, Beijing 100871, China. Email address: yangjp@math.pku.edu.cn 
See Joe (1997) and Nelsen (2006) for more introduction about copula functions. In fact, now copula method becomes more and more important in quantitative finance and risk management, see McNeil et al. (2005) and Denuit et al. (2005).

Gaussian copula and Archimedean copula are important copula families applied in finance and insurance. Proposed by Li (2000), Gaussian copula has been widely used in credit risk modeling, and it is also criticized after the subprime mortgage crisis for its incapacity to capture high correlation in tails (Donnelly and Embrechts, 2010). As an alternative for Gaussian copula, Student T-copula is capable of capturing high correlation in tails, but unfortunately Student T-copula can not describe the asymmetry between the lower and upper tails. In high-dimensional case, Archimedean copula is very popular for its explicit expression and its convenience for sampling. However, most of the Archimedean families have few parameters, which makes the copulas exchangeable and less flexible. Therefore, it will be important to construct copula functions sharing the good properties of several copula families.

Fundamental transformation-based copula construction methods include convex sum, ordinal sum and shuffle of copula (Nelsen, 2006). Recently, the distortion function has been applied for constructing copula functions. A continuous function $D(x)$ from $[0,1]$ to $[0,1]$ is called a distortion function if $D$ is increasing and $D(0)=0, D(1)=1$. Yaari (1987) firstly applied the distortion function in dual theory of choice under risk, and Wang (1996) defined Wang's premium principle by using distortion functions. Genest and Rivest (2001), Klement et al. (2005), Durante and Sempi (2005) and Durante et al. (2010) considered transformations from a bivariate copula $C\left(u_{1}, u_{2}\right)$ to another one $C_{\varphi}=\varphi^{-1}\left(C\left(\varphi\left(u_{1}\right), \varphi\left(u_{2}\right)\right)\right)$, where $\varphi$ is a distortion function. Morillas (2005) extended this idea to multivariate copulas. In addition, Liebscher (2008) presented two general construction schemes based on product of copulas and generalized Archimedean family. Fischer and Köck (2012) unified the above transformation-based methods.

In this paper, we will introduce a method called Distorted Mix Method (DMM) to construct copulas by applying distortion functions. The idea of DMM is based on the convex sum method, while the copula margins are modified by some distortion functions. By applying the distortion functions, we can model dependence structure by handling central and tail parts separately. More precisely, DMM enables us to modify the tail parts of a given copula to any desired pattern. Our theoretical discussion will focus on two tail dependence measures: tail dependence coefficients of marginal distributions and tail dependence function defined in Klüppelberg et al. (2008) and Joe et al. (2010). Through choosing suitable distortion functions, DMM can be applied to construct copulas being close to a given copula, and its tail dependence can reach any desired level. Empirical results will also be given to show that a modified Gaussian copula constructed by DMM performs significantly better than Gaussian copulas in the tail parts.

This paper is organized as follows. In Section 2, we introduce our definition of DMM, and theoretical results about tail behavior of the constructed copulas are provided. In Section 3, we apply DMM to change tail dependence of a given copula, in which we focus on two tail measures: tail dependence function and tail dependence coefficients of marginal distributions. Empirical results are presented in Section 4 and conclusion is given in Section 5. Some proofs are provided in the appendix. 


\section{Distorted Mix Method}

\subsection{Definition for Distorted Mix Method}

Given integer $m \geq 2$ and the corresponding weights $\alpha_{i}>0, i=1,2, \ldots, m$ with $\sum_{i=1}^{m} \alpha_{i}=1$, let the distortion functions $D_{i j}, i=1, \ldots, m, j=1, \ldots, d$ satisfy the following assumption:

- Assumption A: $\sum_{i=1}^{m} \alpha_{i} D_{i j}(x)=x$ for any $j=1, \ldots, d$.

Then based on copula functions $C_{i}, i=1, \ldots, m$ and the distortion functions $D_{i j}, i=1, \ldots, m, j=$ $1, \ldots, d$, we define a function $\tilde{C}\left(u_{1}, \ldots, u_{d}\right)$ as

$$
\tilde{C}\left(u_{1}, \ldots, u_{d}\right)=\sum_{i=1}^{m} \alpha_{i} \cdot C_{i}\left(D_{i 1}\left(u_{1}\right), \ldots, D_{i d}\left(u_{d}\right)\right)
$$

The above definition combines the ideas of distortion and convex sum, so we name this method Distorted Mix Method (DMM).

Next theorem will show that the function $\tilde{C}\left(u_{1}, \ldots, u_{d}\right)$ is a copula function under Assumption A, regardless of the choice of copulas, distortion functions and corresponding weights. The proof will be given in the appendix.

Theorem 1. Assume that Assumption A holds. Then the function $\tilde{C}\left(u_{1}, \ldots, u_{d}\right)$ defined in (1) is a copula function. Moreover, for $i=1, \ldots, m$,

$$
\sup _{\left(u_{1}, \ldots, u_{d}\right) \in[0,1]^{d}}\left|\tilde{C}\left(u_{1}, \ldots, u_{d}\right)-C_{i}\left(u_{1}, \ldots, u_{d}\right)\right| \leq \min \left\{1,\left(1-\alpha_{i}\right)(d+1)\right\}
$$

and

$$
\int_{[0,1]^{d}}\left|\tilde{C}\left(u_{1}, \ldots, u_{d}\right)-C_{i}\left(u_{1}, \ldots, u_{d}\right)\right| d u_{1} \ldots d u_{d} \leq \min \left\{1,\left(1-\alpha_{i}\right)(d / 2+1)\right\} .
$$

In the next we call the copula $\tilde{C}$ a DM copula, and we call copulas $C_{i}, i=1, \ldots, m$ the component copulas. Essentially, a DM copula is a mixture of different component copulas with margins modified by some distortion functions. Notice that for each fixed $i$, the choice of the component copula $C_{i}$ is irrelevant to the choice of weight $\alpha_{i}$ and corresponding distortion functions $D_{i j}, j=1, \ldots, d$.

Intuitively, as the weight $\alpha_{i}$ gets larger, the DM copula $\tilde{C}$ will get closer to the component copula $C_{i}$. The above inequalities (2) and (3) show that the DM copula $\tilde{C}$ is close to $C_{i}$ when $\alpha_{i}$ is close to 1 .

We can explain DM copula from the following two viewpoints. The first is that DMM is a generalization of the convex sum method. Letting $D_{i j}(u)=u, \tilde{C}\left(u_{1}, \ldots, u_{d}\right)$ can be expressed as a convex sum

$$
\tilde{C}\left(u_{1}, \ldots, u_{d}\right)=\sum_{i=1}^{m} \alpha_{i} \cdot C_{i}\left(u_{1}, \ldots, u_{d}\right) .
$$

Compared with the convex sum method, DMM uses a technique of distortion to make each component copula focus on a particular aspect. The second viewpoint is that DM copula can be derived 
from convex sum of distribution functions. Let $H$ and $H_{i}$ be $d$-dimensional continuous distributions satisfying

$$
H\left(x_{1}, \ldots, x_{d}\right)=\sum_{i=1}^{m} \alpha_{i} \cdot H_{i}\left(x_{1}, \ldots, x_{d}\right) .
$$

Note that $H$ and $H_{i}$ can also be expressed as

$$
H\left(x_{1}, \ldots, x_{d}\right)=C\left(F_{1}\left(x_{1}\right), \ldots, F_{d}\left(x_{d}\right)\right), H_{i}\left(x_{1}, \ldots, x_{d}\right)=C_{i}\left(F_{i 1}\left(x_{1}\right), \ldots, F_{i d}\left(x_{d}\right)\right),
$$

where $C, C_{i}$ are copula functions, and $F_{i}, F_{i j}$ are marginal distributions. Then the above copula function $C$ can be written as

$$
C\left(u_{1}, \ldots, u_{d}\right)=\sum_{i=1}^{m} \alpha_{i} \cdot C_{i}\left(F_{i 1}\left(F_{1}^{-1}\left(u_{1}\right)\right), \ldots, F_{i d}\left(F_{d}^{-1}\left(u_{d}\right)\right)\right),
$$

which shows that the distortion function $D_{i j}$ can be regarded as a transformation between the margins

$$
D_{i j}(x)=F_{i j}\left(F_{j}^{-1}(x)\right) .
$$

In the next, we provide a factor illustration to explain DMM from the view of random variables. Based on this view, we can get a better understanding of DM copula and its distortion functions.

Proposition 1. Consider a common factor $Z$ distributing discretely as $\mathbb{P}(Z=i)=\alpha_{i}$ for $i=$ $1, \ldots, m$. For each fixed $i$, the random variables $U_{1}, \ldots, U_{d}$ satisfy that $\mathbb{P}\left(U_{j} \leq x \mid Z=i\right)=D_{i j}(x)$, and their conditional copula under $Z=i$ is denoted as $C_{i}\left(u_{1}, \ldots, u_{d}\right)$. Then if Assumption $\mathbf{A}$ holds, the joint distribution of $U_{1}, \ldots, U_{d}$ is the DM copula defined in (1).

The proof of the proposition is obvious and omitted. The above proposition provides a clear probability structure for DM copulas. It shows that DM copulas can describe different dependence structures in different market circumstances, which is quite reasonable in finance (Longin and Solnik, 2001).

Owing to the factor illustration of DM copulas, the procedure of sampling a DM copula can be divided into two parts: first sample each component copula $C_{i}$, then compute the inverse functions $D_{i j}^{-1}$. More precisely, to sample a random vector $\mathbf{U}=\left(U_{1}, \ldots, U_{d}\right)$ whose joint distribution is $\tilde{C}$ defined in equation (1), we can work on it according to the following steps:

1. Sample a random variable $Z$ distributing discretely as $\mathbb{P}(Z=i)=\alpha_{i}$ for $i=1, \ldots, m$;

2. Sample a random vector $\mathbf{V}=\left(V_{1}, \ldots, V_{d}\right)$ whose joint distribution function is $C_{Z}$;

3. Let $U_{j}=D_{Z j}^{-1}\left(V_{j}\right)$.

Then $\mathbf{U}=\left(U_{1}, \ldots, U_{d}\right)$ is a sample of $\tilde{C}$. This algorithm provides a sampling scheme based on each component copula, which avoids sampling directly based on the expression of $\tilde{C}$. Therefore, if all the component copulas are easy to sample, it will also be easy to sample the DM copula.

Besides the advantage in simulation, the DM copula inherits many good properties from its component copulas. For instance, if all the component copulas $C_{i}$ and the distortion functions $D_{i j}$ have continuous density functions, then the DM copula also has a continuous density function.

In most cases of the following discussion, we assume that the distortion functions in the same component copula are identical. Precisely, let $D_{i}:=D_{i 1}=D_{i 2}=\ldots=D_{i d}$ for each $i$, then Assumption $\mathbf{A}$ is simplified as 
- Assumption B: $\sum_{i=1}^{m} \alpha_{i} D_{i}(x)=x$.

Under Assumption B, the DM copula is simplified as

$$
\tilde{C}\left(u_{1}, \ldots, u_{d}\right)=\sum_{i=1}^{m} \alpha_{i} \cdot C_{i}\left(D_{i}\left(u_{1}\right), \ldots, D_{i}\left(u_{d}\right)\right) .
$$

Remark 1. If the distortion functions in the same vector component are identical, i.e., $D_{1 j}=D_{2 j}=$ $\ldots=D_{m j}$ for all $j$, then Assumption A implies that all the distortion functions are simply reduced to $D_{i j}(x)=x$. In this case, the DM copula becomes the convex sum of the component copulas.

\subsection{Examples of DM copulas}

As is well known, Gaussian copula can not describe heavy tail correlation of financial variables. Next we construct a more flexible copula family by using DMM. The intuitive idea is to modify a Gaussian copula into a new copula with Archimedean tails.

Example 1. Let $C_{1}$ be a $d$-dimensional Gaussian copula $C_{\Sigma}^{N}$ with correlation matrix $\Sigma, C_{2}$ be a Clayton copula $C_{\theta}^{C l}$ and $C_{3}$ be a Gumbel copula $C_{\psi}^{G u}$, i.e.,

$$
C_{\theta}^{C l}\left(u_{1}, \ldots, u_{d}\right)=\left(\sum_{j=1}^{d} u_{j}^{-\theta}-d+1\right)^{-\frac{1}{\theta}}, \quad C_{\psi}^{G u}\left(u_{1}, \ldots, u_{d}\right)=\exp \left(-\left[\sum_{j=1}^{d}\left(-\ln u_{j}\right)^{\psi}\right]^{\frac{1}{\psi}}\right)
$$

and

$$
C_{\Sigma}^{N}\left(u_{1}, \ldots, u_{d}\right)=\Phi_{\Sigma}\left(\Phi^{-1}\left(u_{1}\right), \ldots, \Phi^{-1}\left(u_{d}\right)\right),
$$

where $\theta \in(0, \infty), \psi \in[1, \infty), \Phi$ is the univariate Gaussian distribution function and $\Phi_{\Sigma}$ is the multivariate Gaussian distribution function with correlation matrix $\Sigma$.

Let $\alpha \in\left(0, \frac{1}{2}\right), \alpha_{1}=1-2 \alpha, \alpha_{2}=\alpha_{3}=\alpha$, and choose the distortion functions

$$
D_{2}(x)=\frac{x-\alpha x^{2}}{\alpha+(1-2 \alpha) x}, D_{3}(x)=\frac{\alpha x^{2}}{\alpha+(1-2 \alpha)(1-x)}, D_{1}(x)=\frac{x-\alpha D_{2}(x)-\alpha D_{3}(x)}{1-2 \alpha} .
$$

It is easy to check $\sum_{i=1}^{3} \alpha_{i} D_{i}(x)=x$, so Assumption $\mathbf{B}$ holds. According to Theorem 1, the DM copula defined in (4) is close to the Gaussian copula $C_{\Sigma}^{N}$ when $\alpha$ is small. Furthermore, the numerical results in Figure 2 show that the DM copula performs like a Clayton copula in the lower tail, and like a Gumbel copula in the upper tail. Here we only provide an intuitive explanation, and the theoretical results will be given in the next subsection.

For the above DM copula, we consider its factor illustration in Proposition 1. Suppose there is a common factor $Z$ taking values in $\{1,2,3\}$. According to Proposition 1 , let $\left(U_{1}, \ldots, U_{d}\right)$ be a sample of the DM copula, then the conditional distribution functions of $U_{j}, j=1, \ldots, d$ under $Z=i$ are all equal to $D_{i}(x)$. Figure 1 shows the conditional distribution functions $D_{1}, D_{2}, D_{3}$ and their density functions $D_{1}^{\prime}, D_{2}^{\prime}, D_{3}^{\prime}$ when $\alpha=0.02$. The density functions $D_{2}^{\prime}, D_{3}^{\prime}$ imply that $U_{j}, j=1, \ldots, d$ are more likely to take small values under $Z=2$ and take large values under $Z=3$. On the other hand, Proposition 1 says that the conditional copula of $U_{1}, \ldots, U_{d}$ under $Z=2$ is the 
(a) $D_{1}(x), D_{2}(x), D_{3}(x)$

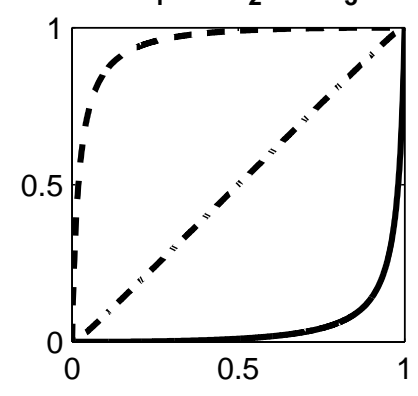

(b) Densities of $D_{2}(x), D_{3}(x)$

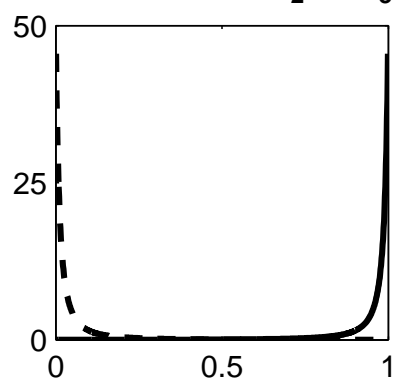

(c) Density of $D_{1}(x)$

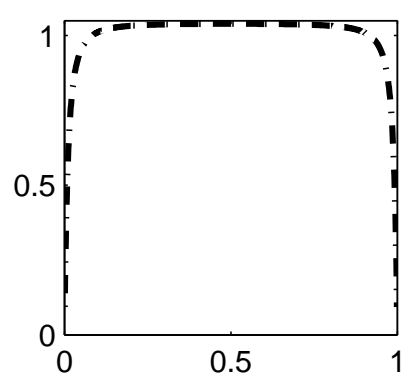

Figure 1: Subplot (a) shows the distortion functions $D_{1}(x), D_{2}(x), D_{3}(x)$ defined in (5) when $\alpha=0.02$. Subplot (b) and (c) show the density functions $D_{1}^{\prime}, D_{2}^{\prime}, D_{3}^{\prime}$ when $\alpha=0.02$. The dash dotted, dashed and solid lines are $D_{1}, D_{2}, D_{3}$ and their density functions respectively.

Clayton copula $C_{\theta}^{C l}$, so the lower tail of the DM copula performs like a Clayton copula. Similarly, the upper tail of the DM copula performs like a Gumbel copula.

Figure 2 shows density contours of bivariate meta-distributions with different bivariate copulas and standard Gaussian margins. The DM copulas in Figure 2(d)-Figure 2(f) are defined in Example 1. Here the component Gaussian copula $C_{\rho}^{N}$ has correlation $\rho=0.3$, the component Clayton copula $C_{\theta}^{C l}$ has $\theta=0.7565$, and the component Gumbel copula $C_{\psi}^{G u}$ has $\psi=1.7095$. Figure 2(g)-Figure 2(i) display the cases of convex sum copula

$$
C^{C s}\left(u_{1}, u_{2}\right)=(1-2 \alpha) \cdot C_{\rho}^{N}\left(u_{1}, u_{2}\right)+\alpha \cdot C_{\theta}^{C l}\left(u_{1}, u_{2}\right)+\alpha \cdot C_{\psi}^{G u}\left(u_{1}, u_{2}\right) .
$$

Joe-Clayton copula in Figure 2(c) is defined in Joe (1997) as

$$
C^{J c}(u, v)=1-\left\{1-\left[\left(1-(1-u)^{\psi}\right)^{-\theta}+\left(1-(1-v)^{\psi}\right)^{-\theta}-1\right]^{-1 / \theta}\right\}^{1 / \psi},
$$

in which $(\theta, \psi) \in(0, \infty) \times[1, \infty)$. Here we choose $\theta=0.7565$ and $\psi=1.7095$ as above.

Compared with the convex sum copulas, the DM copulas show greater flexibility. When $\alpha=$ 0.02 in Figure 2(d), the weight $\alpha_{1}$ of the component Gaussian copula is 0.96 . Since $\alpha_{1}$ is close to 1 , the DM copula is similar to the Gaussian copula, hence the contours in Figure 2(d) look like the Gaussian density contours in Figure 2(a). However, the DM copula performs like a Clayton copula in the lower tail and like a Gumbel copula in the upper tail, so the tail contours are sharper than those of Gaussian density. As $\alpha$ increases, the weight of component Gaussian copula decreases, and the density contours turn to be spindle-shaped. It is interesting that the density contours with $\alpha=0.25$ in Figure 2(f) are remarkably similar to those of the Joe-Clayton copula in Figure 2(c).

In summary, this example shows roughly that DMM enables us to construct copulas by choosing central and tail parts separately.

\subsection{Tail dependence modification}

Tail dependence coefficient (TDC) is the most popular measure of tail correlation. For a $d$-dimensional copula $C$, its lower TDC is defined as $\lambda_{C}=\lim _{u \downarrow} C(u, \ldots, u) / u$, provided the 
(a) Gaussian copula $(\rho=0.3)$

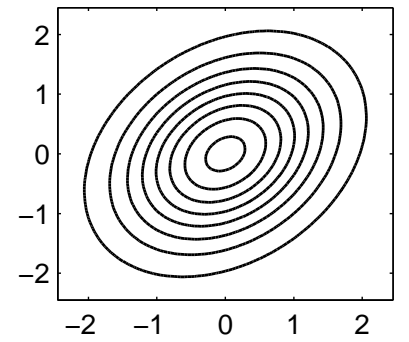

(d) DM Copula $(\alpha=0.02)$

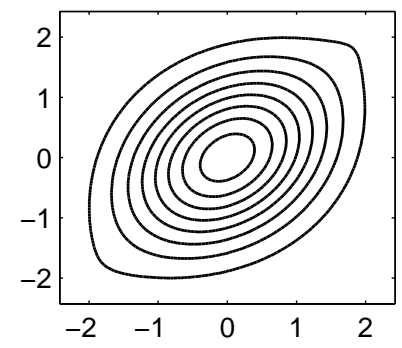

(g) Convex Sum $(\alpha=0.02)$

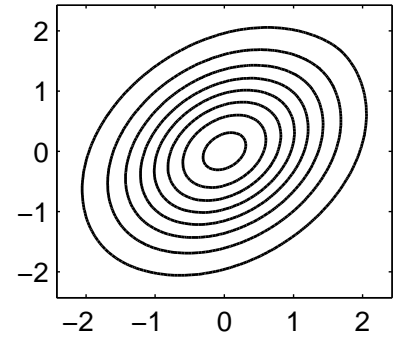

(b) T-copula $(\rho=0.3, v=5)$

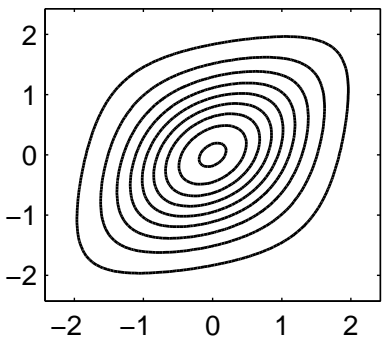

(e) DM Copula $(\alpha=0.05)$

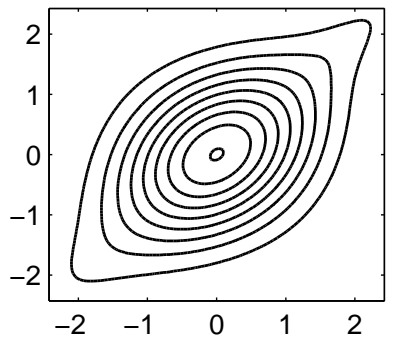

(h) Convex Sum $(\alpha=0.05)$

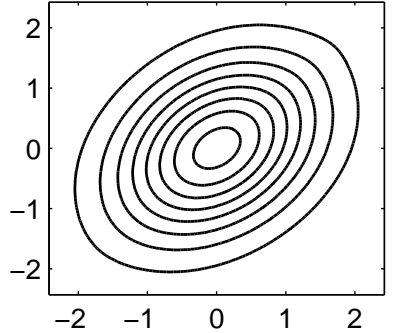

(c) Joe-Clayton Copula

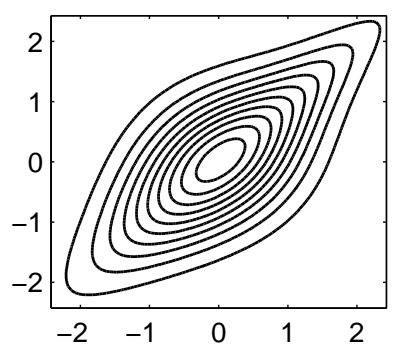

(f) DM Copula $(\alpha=0.25)$

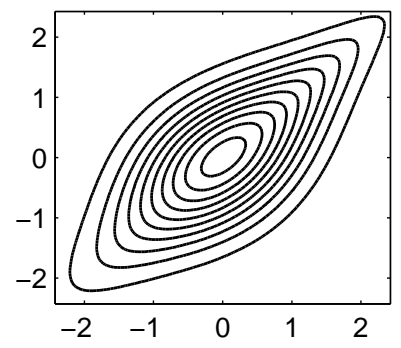

(i) Convex Sum $(\alpha=0.25)$

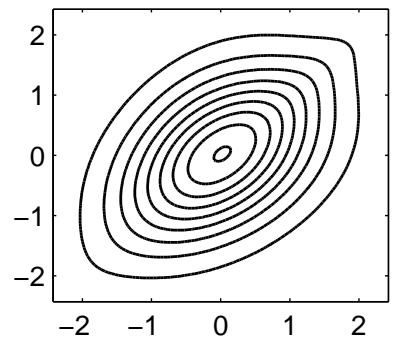

Figure 2: Density contours of meta-distributions with nine different bivariate copulas and standard Gaussian margins. DM copulas are mixed by distorted Gaussian, Clayton and Gumbel copula with different $\alpha$ in Example 1.

limit exists. And the upper TDC is defined as the lower TDC of its survival copula, i.e., $\lambda_{C}^{*}=$ $\lim _{u \downarrow 0} \hat{C}(u, \ldots, u) / u$. Here the survival copula $\hat{C}$ of copula function $C$ is defined as

$$
\hat{C}\left(u_{1}, u_{2}, \ldots, u_{d}\right)=\mathbb{P}\left(U_{1} \geq 1-u_{1}, \ldots, U_{d} \geq 1-u_{d}\right),
$$

where $\mathbf{U}=\left(U_{1}, \ldots, U_{d}\right)$ is a sample of $C$. Both the lower TDC and the upper TDC are in [0,1], and large TDC corresponds to strong correlation in tail parts.

The concept of TDC can be extended to the concept of tail dependence function (TDF) defined by Klüppelberg et al. (2008). Its copula version has been introduced by Joe et al. (2010) as following. The lower TDF is defined as

$$
\left.b\left(w_{1}, \ldots, w_{d} ; C\right)=\lim _{u \downarrow 0} C\left(w_{1} u, \ldots, w_{d} u\right)\right) / u,
$$

and the upper TDF is defined as

$$
b^{*}\left(w_{1}, \ldots, w_{d} ; C\right)=\lim _{u \downarrow 0} \hat{C}\left(w_{1} u, \ldots, w_{d} u\right) / u,
$$


in which $\left(w_{1}, \ldots, w_{d}\right) \in[0, \infty)^{d}$. Notice that $b(1, \ldots, 1 ; C)=\lambda_{C}$ and $b^{*}(1, \ldots, 1 ; C)=\lambda_{C}^{*}$. Unless specially stated, we assume TDC and TDF always exist afterwards.

Next we extend Example 1 to a more general case. Our purpose is to replace the TDF of a given copula, say $C_{1}$. Assuming that we have a copula $C_{2}$ at hand, we prefer the TDF of $C_{2}$ to that of $C_{1}$. For this purpose we will construct a copula function $\tilde{C}$ close to $C_{1}$ and its TDF is identical to that of $C_{2}$.

Proposition 2. Assume that $C_{1}, C_{2}$ are copulas and $\alpha_{1}, \alpha_{2}, D_{1}, D_{2}$ satisfy Assumption $\mathbf{B}$, then the distance between the DM copula

$$
\tilde{C}\left(u_{1}, \ldots, u_{d}\right)=\alpha_{1} C_{1}\left(D_{1}\left(u_{1}\right), \ldots, D_{1}\left(u_{d}\right)\right)+\alpha_{2} C_{2}\left(D_{2}\left(u_{1}\right), \ldots, D_{2}\left(u_{d}\right)\right)
$$

and $C_{1}$ can be measured by (2) and (3) for $i=1$. Furthermore,

(I) if $\alpha_{2} D_{2}^{\prime}(0+)=1$, the DM copula $\tilde{C}$ and copula $C_{2}$ have identical lower TDF;

(II) if $\alpha_{2} D_{2}^{\prime}(1-)=1$, the DM copula $\tilde{C}$ and copula $C_{2}$ have identical upper TDF.

Proof. Here we only prove the lower tail case in part (I), and the proof of the part (II) is similar.

For the $d$-dimensional copulas $C_{i}$, if $D_{i}^{\prime}(0+)$ exists,

$$
\begin{aligned}
\lim _{u \downarrow 0} \frac{C_{i}\left(w_{1} D_{i}(u), \ldots, w_{d} D_{i}(u)\right)}{u} & =\lim _{u \downarrow 0} \frac{C_{i}\left(w_{1} D_{i}(u), \ldots, w_{d} D_{i}(u)\right)}{D_{i}(u)} \cdot \frac{D_{i}(u)}{u} \\
& =D_{i}^{\prime}(0+) \cdot b\left(w_{1}, \ldots, w_{d} ; C_{i}\right) .
\end{aligned}
$$

Owing to the Lipchitz property of copula function, for a small $u$ we have

$$
\left|C_{i}\left(w_{1} D_{i}(u), \ldots, w_{d} D_{i}(u)\right)-C_{i}\left(D_{i}\left(w_{1} u\right), \ldots, D_{i}\left(w_{d} u\right)\right)\right| \leq \sum_{j=1}^{d}\left|w_{j} D_{i}(u)-D_{i}\left(w_{j} u\right)\right|=o(u) .
$$

By integrating the above two aspects, we have

$$
\lim _{u \downarrow 0} \frac{C_{i}\left(D_{i}\left(w_{1} u\right), \ldots, D_{i}\left(w_{d} u\right)\right)}{u}=D_{i}^{\prime}(0+) \cdot b\left(w_{1}, \ldots, w_{d} ; C_{i}\right), \forall\left(w_{1}, \ldots, w_{d}\right) \in[0, \infty)^{d} .
$$

Now we go back to the proof of Proposition 2. From $\alpha_{1} D_{1}(x)+\alpha_{2} D_{2}(x)=x$ we know that $\alpha_{1} D_{1}^{\prime}(0+)=0$. Applying (8), we have

$$
\begin{aligned}
b\left(w_{1}, \ldots, w_{d} ; \tilde{C}\right) & =\alpha_{1} D_{1}^{\prime}(0+) \cdot b\left(w_{1}, \ldots, w_{d} ; C_{1}\right)+\alpha_{2} D_{2}^{\prime}(0+) \cdot b\left(w_{1}, \ldots, w_{d} ; C_{2}\right) \\
& =b\left(w_{1}, \ldots, w_{d} ; C_{2}\right)
\end{aligned}
$$

for any $\left(w_{1}, \ldots, w_{d}\right) \in[0, \infty)^{d}$.

Another idea is using other two different copulas to replace the lower and upper TDF respectively, which makes the lower and upper TDF belong to different types.

Proposition 3. Assume $C_{i}, i=1,2,3$ are copulas and $\alpha_{i}, D_{i}, i=1,2,3$ satisfy Assumption $\mathbf{B}$, then the distance between the DM copula

$$
\tilde{C}\left(u_{1}, \ldots, u_{d}\right)=\sum_{i=1}^{3} \alpha_{i} C_{i}\left(D_{i}\left(u_{1}\right), \ldots, D_{i}\left(u_{d}\right)\right)
$$

and $C_{1}$ can be measured by (2) and (3) for $i=1$. Furthermore, if $\alpha_{2} D_{2}^{\prime}(0+)=\alpha_{3} D_{3}^{\prime}(1-)=1$, the DM copula $\tilde{C}$ has identical lower TDF with $C_{2}$ and identical upper TDF with $C_{3}$. 
The proof is similar and omitted. Next we provide three distortion function families

$$
H_{\alpha}^{p}(x)=x^{1 / \alpha}, \quad H_{\alpha}^{e}(x)=x^{2} e^{(1 / \alpha-2)(x-1)}, \quad H_{\alpha, \beta}^{f}(x)=\frac{\alpha \beta x^{2}}{\alpha \beta+(1-2 \alpha)\left(1-x^{\beta}\right)},
$$

where $\alpha, \beta>0$. These distortion functions all have continuous derivatives on $[0,1]$, and each of them satisfies $H^{\prime}(1)=1 / \alpha$ and $H^{\prime}(0)=0$. Notice that if $H(x)$ is a distortion function, then $G(x)=1-H(1-x)$ is also a distortion function satisfying $G^{\prime}(1)=H^{\prime}(0)$ and $G^{\prime}(0)=H^{\prime}(1)$. Thus if we choose $D_{2}(x)=1-(1-x)^{1 / \alpha_{2}}$ for example, then $\alpha_{2} D_{2}^{\prime}(0+)=1$. The above candidates of distortion functions satisfy the conditions in Proposition 2 and Proposition 3.

Example 1 (continued). The distortion function in Example 1 is the case $1-D_{2}(1-x)=D_{3}(x)=$ $H_{\alpha, 1}^{f}(x)$, so the DM copulas $\tilde{C}$ in Example 1 have identical lower TDF with Clayton copula and identical upper TDF with Gumbel copula. Hence its lower and upper TDC satisfies

$$
\left(\lambda_{\tilde{C}}, \lambda_{\tilde{C}}^{*}\right)=\left(2^{-1 / \theta}, 2-2^{1 / \psi}\right), \quad(\theta, \psi) \in(0, \infty) \times[1, \infty)
$$

which implies $\left(\lambda_{\tilde{C}}, \lambda_{\tilde{C}}^{*}\right)$ can vary freely in $(0,1)^{2}$. Thus all the DM copulas in Figure 2(d)-Figure 2 (f) have the same lower TDC $2^{-1 / 0.7565}=0.4$ and the same upper TDC $2-2^{1 / 1.7095}=0.5$.

Remark 2. From the discussion above, we can confirm the advantage of DMM over the convex sum method. Although convex sum is widely used as a tool in time series empirical study (Chen and Fan, 2006), the convex sum copula is incapable of letting its lower and upper TDC vary freely in $[0,1]^{2}$. More precisely, for the convex sum copula $C^{C s}$ defined in equation (6), we have $\lambda_{C} \leq s \leq \alpha_{2}$ and $\lambda_{C^{C s}}^{*} \leq \alpha_{3}$, thus $\lambda_{C^{C s}}+\lambda_{C^{C s}}^{*} \leq 1$ follows.

\subsection{On $\alpha_{i}$ 's selection}

In this subsection, we discuss on how to select the weights $\alpha_{1}, \ldots, \alpha_{m}$. For simplicity, we will focus on the DM copula defined in the following form

$$
\tilde{C}\left(u_{1}, \ldots, u_{d}\right)=\sum_{i=1}^{3} \alpha_{i} C_{i}\left(D_{i}\left(u_{1}\right), \ldots, D_{i}\left(u_{d}\right)\right),
$$

where the component copula $C_{1}$ is intentionally chosen to capture the central part, and the copulas $C_{2}$ and $C_{3}$ are chosen to capture the lower tail part and the upper tail part separately. In practice, the copula $C_{1}$ may belong to some commonly used copula family with improper tails, for example Gaussian copulas, and $C_{2}, C_{3}$ belong to some copula families whose lower/upper TDC can vary freely in $[0,1]$. As mentioned in Proposition 3, the DM copula in (11) can be applied to modify the tail parts of copula $C_{1}$ into the patterns of $C_{2}$ and $C_{3}$.

By the definition of DM copula, the distortion functions $D_{1}(u), D_{2}(u), D_{3}(u)$ and the weights $\alpha_{1}, \alpha_{2}, \alpha_{3} \geq 0$ with $\alpha_{1}+\alpha_{2}+\alpha_{3}=1$ must satisfy the following condition

$$
\alpha_{1} D_{1}(u)+\alpha_{2} D_{2}(u)+\alpha_{3} D_{3}(u)=u, \quad \forall u \in[0,1]
$$

On the other hand, the condition

$$
\alpha_{2} D_{2}^{\prime}(0+)=\alpha_{3} D_{3}^{\prime}(1-)=1
$$


is required to guarantee that the DM copula $\tilde{C}$ has identical lower TDF with $C_{2}$ and identical upper TDF with $C_{3}$. Under the constraints of (12) and (13), the distortion functions $D_{1}(u), D_{2}(u), D_{3}(u)$ are related to the parameters $\alpha_{1}, \alpha_{2}$ and $\alpha_{3}$. The distortion functions can be chosen from some parametric families. Some distortion functions belonging to the power, exponential and fractional families are provided in (10), in which the distortion functions are fully or partially determined by the weight parameters. More generally, we can get other parametric distortion families by the following method:

- Choose two parametric positive continuous functions $h_{2}, h_{3}$ with domain $[0,1]$ satisfying $h_{2}(x)+h_{3}(x) \leq 1, x \in[0,1]$ and

$$
\begin{aligned}
& h_{2}(0)=1, h_{2}(1)=0, \int_{0}^{1} h_{2}(x) d x=\alpha_{2}, \\
& h_{3}(0)=0, h_{3}(1)=1, \int_{0}^{1} h_{3}(x) d x=\alpha_{3} ;
\end{aligned}
$$

- Set

$$
D_{2}(u)=\int_{0}^{u} \frac{h_{2}(x)}{\alpha_{2}} d x, \quad D_{3}(u)=\int_{0}^{u} \frac{h_{3}(x)}{\alpha_{3}} d x, \quad D_{1}(u)=\int_{0}^{u} \frac{1-h_{2}(x)-h_{3}(x)}{1-\alpha_{2}-\alpha_{3}} d x,
$$

then $D_{1}(u), D_{2}(u), D_{3}(u)$ are distortion functions satisfying (12) and (13).

In the next, we discuss on how to estimate the parameters $\alpha_{1}, \alpha_{2}$ and $\alpha_{3}$.

For the purpose of our DM method, $\tilde{C}$ should be close to copula $C_{1}$ and have the same tail patterns as $C_{2}$ and $C_{3}$. Note that when (13) holds, the lower and upper TDFs of the DM copula $\tilde{C}$ are fully determined by the component copulas $C_{2}, C_{3}$ and independent to the values of the weights $\alpha_{1}, \alpha_{2}, \alpha_{3}$. On the other hand, the difference between the DM copula $\tilde{C}$ and its component copula $C_{1}$ is highly related to the value of $1-\alpha_{1}$. Precisely, from (2) we see that

$$
\sup _{\left(u_{1}, \ldots, u_{d}\right) \in[0,1]^{d}}\left|\tilde{C}\left(u_{1}, \ldots, u_{d}\right)-C_{1}\left(u_{1}, \ldots, u_{d}\right)\right| \leq\left(1-\alpha_{1}\right)(d+1) .
$$

Thus in order that $\tilde{C}$ and $C_{1}$ are close enough, one requirement is that $1-\alpha_{1}=\alpha_{2}+\alpha_{3}$ is small. Therefore, we can consider estimating $\alpha_{2}, \alpha_{3}$ in a small range, say $[0, \beta]$. Thus, with given parametric distortion functions $D_{1}, D_{2}$ and $D_{3}$ satisfying (12) and (13), statistical estimation can be applied to estimate $\alpha_{2}, \alpha_{3} \in[0, \beta]$ and other parameters.

The estimation of the DM copula can be implemented by the following procedure:

- Step 1: Use a parametric copula family to fit the data and estimate the parameters. Denote $C_{1}$ as the fitted copula. If the copula $C_{1}$ doesn't fit the tail parts very well, we do the following steps to modify $C_{1}$ by DMM.

- Step 2: Choose the families of the component copulas $C_{2}, C_{3}$ and the distortion functions $D_{1}, D_{2}$ and $D_{3}$ to model the tail parts. Note that $\alpha_{1}+\alpha_{2}+\alpha_{3}=1$, and the distortion functions are also related to $\alpha_{2}$ and $\alpha_{3}$. One can set the weights $\alpha_{2}$ and $\alpha_{3}$ as constants, or limit $\alpha_{2}, \alpha_{3}$ in a pre-set range. 
- Step 3: Calibrate the parameters in $C_{2}, C_{3}, D_{1}, D_{2}$ and $D_{3}$ by Maximum Likelihood Estimation (MLE). If the parameters $\alpha_{2}, \alpha_{3}$ are unknown before this step, then the estimates of the two parameters can be obtained in this step.

In Section 4, we will apply the above procedure to analyze financial return data.

\section{Constructing copulas with tail dependence by DMM}

In this section, we will discuss on modifying a given copula to meet some specific tail requirements by applying DMM. Here we consider two tail measures: tail dependence function (TDF) and tail dependence coefficients (TDC) of marginal distributions. We will only focus on the lower tail in this section, and the results of upper tail are similar.

\subsection{Constructing Copulas with preferred TDF}

In Section 2.3, we used DMM to replace the TDF of a given copula. Of course doing such a procedure has a certain premise that we have already chosen the alternative copula. If there is no candidate, we can focus on TDF directly. In the following, we will discuss on constructing copula functions with the objective TDF by DMM.

Joe et al. (2010) has discussed the properties of a TDF. They proved that a TDF $b\left(w_{1}, \ldots, w_{d} ; C\right)$ must be a grounded $d$-increasing function with homogeneity of order 1 . Precisely,

1. (grounded) $b\left(w_{1}, \ldots, w_{d} ; C\right)=0$ if there exists some $w_{i}=0$;

2. $\left(d\right.$-increasing) $\sum_{i_{1}=1}^{2} \ldots \sum_{i_{d}=1}^{2}(-1)^{i_{1}+\ldots+i_{d}} b\left(w_{1}^{\left(i_{1}\right)}, \ldots, w_{d}^{\left(i_{d}\right)} ; C\right) \geq 0$ for $w_{i}^{(1)} \leq w_{i}^{(2)}, i=1, \ldots, d$;

3. (homogeneity of order 1) $b\left(\lambda w_{1}, \ldots, \lambda w_{d} ; C\right)=\lambda \cdot b\left(w_{1}, \ldots, w_{d} ; C\right)$ for any $\lambda \geq 0$.

Next we will give the necessary and sufficient conditions for a function being a TDF, then we will construct copulas with any given TDF by DMM.

Theorem 2. (I) Function B is a TDF, i.e., there exists a d-dimensional copula whose TDF equals $B$, if and only if $B$ satisfies all the following conditions:

(a) $B$ is a grounded and d-increasing function with homogeneity of order 1 .

(b) B is Lipschitz continuous with parameter 1, i.e., $\left|B\left(w_{1}, \ldots, w_{d}\right)-B\left(v_{1}, \ldots, v_{d}\right)\right| \leq \sum_{i=1}^{d}\left|w_{i}-v_{i}\right|$. (II) Assume that function $B$ is a TDF. For $\left(u_{1}, \ldots, u_{d}\right) \in[0,1]^{d}$, we denote

$$
C^{B}\left(u_{1}, \ldots, u_{d}\right)= \begin{cases}B\left(u_{1}, \ldots, u_{d}\right), & \text { if } B(1, \ldots, 1)=1 \\ B\left(u_{1}, \ldots, u_{d}\right)+\frac{\prod_{i=1}^{d}\left(u_{i}-B_{i}\left(u_{i}\right)\right)}{(1-B(1, \ldots, 1))^{d-1}}, & \text { otherwise }\end{cases}
$$

where $B_{i}(x)=\left.B\left(x_{1}, \ldots, x_{d}\right)\right|_{x_{i}=x \& x_{j}=1, \forall j \neq i}$, then $C^{B}\left(u_{1}, \ldots, u_{d}\right)$ in $[0,1]^{d}$ is a copula function. And for any given copula $C_{1}$, the TDF of the DM copula

$$
\tilde{C}\left(u_{1}, u_{2}, \ldots, u_{d}\right)=(1-\alpha) C_{1}\left(D_{1}\left(u_{1}\right), \ldots, D_{1}\left(u_{d}\right)\right)+\alpha C^{B}\left(D_{2}\left(u_{1}\right), \ldots, D_{2}\left(u_{d}\right)\right)
$$

equals $B$ when distortion functions $D_{1}, D_{2}$ satisfy $(1-\alpha) D_{1}(x)+\alpha D_{2}(x)=x$ for $x \in[0,1]$ and $\alpha D_{2}^{\prime}(0+)=1$. Furthermore, the distance between the DM copula $\tilde{C}$ and copula $C_{1}$ can be measured by (2) and (3) for $i=1$. 
Proof. The necessity of property (a) has been proved in Joe et al. (2010). The necessity of Lipschitz property in (b) is inherited from the Lipschitz property of copula function. Next we prove the sufficiency of these properties by constructing copulas with such TDF.

If $B$ is a function satisfying the conditions in (a) and (b), we prove that $C^{B}$ is a copula function. Firstly, we notice that $B$ satisfies the Lipschitz property in (b), which implies $B_{i}(x)-B_{i}(0) \leq x-0$. Hence $B_{i}(x) \leq x$ for any $i=1, \ldots, d$, so we obtain $B(1, \ldots, 1)=B_{1}(1) \leq 1$.

Case 1: $B(1, \ldots, 1)=1$. Then $B_{i}(1)=1$ for $i=1, \ldots, d$. The Lipschitz property implies $B_{i}(1)-B_{i}(x) \leq 1-x$ for $x \in[0,1]$, so $B_{i}(x) \geq x, x \in[0,1]$. On the other hand, we have already proved $B_{i}(x) \leq x$, so $B_{i}(x)=x$ for $x \in[0,1]$ and $i=1, \ldots, d$. Therefore, $B$ has uniform margins in $[0,1]$. Combining with the assumption that $B$ is $d$-increasing, we know $B\left(u_{1}, \ldots, u_{d}\right),\left(u_{1}, \ldots, u_{d}\right) \in$ $[0,1]^{d}$ is a copula function.

Case 2: $B(1, \ldots, 1) \neq 1$. Then $B(1, \ldots, 1)<1$. Owing to the Lipschitz property, for any $0 \leq x \leq y \leq 1$ and $i=1, \ldots, d$, we know $B_{i}(y)-B_{i}(x) \leq y-x$, so $x-B_{i}(x)$ is increasing for $i=1, \ldots, d$. Hence $\prod_{i=1}^{d}\left(u_{i}-B_{i}\left(u_{i}\right)\right)$ is $d$-increasing. Combining with the assumption that $B$ is $d$-increasing, we know $C^{B}$ is also $d$-increasing from (14). And it is easy to verify that $C^{B}$ has uniform $[0,1]$ margins, so $C^{B}$ is indeed a copula function.

Next we prove that the TDF of copula $C^{B}$ equals $B$. The case $B(1, \ldots, 1)=1$ is obvious. When $B(1, \ldots, 1)<1$, we compute directly based on (14),

$$
B\left(w_{1}, \ldots, w_{d}\right) \leq b\left(w_{1}, \ldots, w_{d} ; C^{B}\right) \leq B\left(w_{1}, \ldots, w_{d}\right)+\lim _{u \downarrow 0} u^{d-1}=B\left(w_{1}, \ldots, w_{d}\right),
$$

so $b\left(w_{1}, \ldots, w_{d} ; C^{B}\right)=B\left(w_{1}, \ldots, w_{d}\right)$. For any given copula $C_{1}$, we use $C^{B}$ as the component copula in Proposition 2, and the conclusion of this theorem is a direct result of Proposition 2.

Remark 3. For any copula $C_{1}$ and tail dependence function $B$, we can apply DMM to construct DM copula $\tilde{C}$ such that $\tilde{C}$ is close enough to $C_{1}$ and has TDF $B$. This result enables us to model dependence structure by handling central and tail parts separately, which enlarges the choice of multivariate copula families for application.

Notice that for any tail dependence function $B$,

$$
B\left(w_{1}, \ldots, w_{d}\right) \leq M\left(w_{1}, \ldots, w_{d}\right):=\min \left\{w_{1}, \ldots, w_{d}\right\} .
$$

As a specific case, $B\left(w_{1}, \ldots, w_{d}\right)=M\left(w_{1}, \ldots, w_{d}\right)$ leads to the concept of tail comonotonicity defined in Hua and Joe (2012). According to Theorem 2, we can use DMM to modify any given copula $C_{1}$ to be tail comonotonic. In this case, the component copula $C^{B}\left(u_{1}, \ldots, u_{d}\right)$ in (15) is $M\left(u_{1}, \ldots, u_{d}\right)$, hence the DM copula

$$
\tilde{C}\left(u_{1}, \ldots, u_{d}\right)=(1-\alpha) \cdot C_{1}\left(D_{1}\left(u_{1}\right), \ldots, D_{1}\left(u_{d}\right)\right)+\alpha \cdot \min \left\{D_{2}\left(u_{1}\right), \ldots, D_{2}\left(u_{d}\right)\right\}
$$

is lower tail comonotonic when $\alpha D_{2}^{\prime}(0+)=1$. Furthermore, the DM copula $\tilde{C}$ is close to $C_{1}$ when $\alpha$ is small.

\subsection{Constructing copulas with preferred marginal TDC family}

In this subsection, we will consider TDC of all the marginal distributions of a copula function. 
To begin with, we give some definitions and notations first. Let $C$ be a $d$-dimensional copula function and $\left(U_{1}, \ldots, U_{d}\right)$ be its sample. Denote $\mathbb{D}=\{1, \ldots, d\}$. For any $S \subseteq \mathbb{D},|S| \geq 2$, we denote $C_{S}$ as the $S$-marginal copula of the copula function $C$, i.e., $C_{S}$ is the distribution function of $\left\{U_{i}, i \in S\right\}$. And we denote $b\left(w_{i}, i \in S ; C_{S}\right)$ as the TDF of the marginal copula $C_{S}$, where we define $b\left(w_{i} ; C_{\{i\}}\right)=w_{i}$ for $i=1, \ldots, d$. From the above definitions, we know that

$$
b\left(w_{i}, i \in S ; C_{S}\right)=\lim _{u \downarrow 0} \mathbb{P}\left(\cap_{i \in S}\left\{U_{i} \leq w_{i} u\right\}\right) / u, \quad \forall S \subseteq \mathbb{D}, S \neq \emptyset .
$$

By the definition of TDC, we know $\lambda_{C_{S}}=b\left(1, \ldots, 1 ; C_{S}\right)$. We call $\left\{\lambda_{C_{S}}: S \subseteq \mathbb{D},|S| \geq 2\right\}$ as the marginal TDC family of the copula $C$.

To consider the compatibility of marginal TDC family, we need to define some more fundamental limits as following

$$
e_{S}\left(w_{1}, \ldots, w_{d} ; C\right)=\lim _{u \downarrow 0} \mathbb{P}\left(\cap_{i \in S}\left\{U_{i} \leq w_{i} u\right\} \cap_{j \notin S}\left\{U_{j}>w_{j} u\right\}\right) / u, \forall S \subseteq \mathbb{D}, S \neq \emptyset .
$$

Comparing (16) with (17) and applying the inclusion and exclusion principle, we can obtain

$$
b\left(w_{i}, i \in S ; C_{S}\right)=\sum_{S \subseteq J \subseteq \mathbb{D}} e_{J}\left(w_{1}, \ldots, w_{d} ; C\right), \quad \forall S \subseteq \mathbb{D}, S \neq \emptyset
$$

and

$$
e_{S}\left(w_{1}, \ldots, w_{d} ; C\right)=\sum_{S \subseteq J \subseteq \mathbb{D}}(-1)^{|J|-|S|} b\left(w_{i}, i \in J ; C_{J}\right), \quad \forall S \subseteq \mathbb{D}, S \neq \emptyset .
$$

Next we provide the necessary and sufficient conditions for a set of numbers $\left\{\beta_{I}: I \subseteq \mathbb{D},|I| \geq\right.$ $2\}$ being a marginal TDC family of a copula function. And we will use DMM to construct copulas with arbitrary compatible TDC family. In fact, we will give a more generalized result through the concept of TDF.

Theorem 3. Fix $\left(w_{1}, \ldots, w_{d}\right) \in[0, \infty)^{d}$.

(I) For a group of numbers $\left\{\beta_{I}: I \subseteq \mathbb{D},|I| \geq 2\right\}$, if there exists a d-dimensional copula $C$ such that $b\left(w_{i}, i \in I ; C_{I}\right)=\beta_{I}$ for all $I \subseteq \mathbb{D},|I| \geq 2$, then

$$
\mu_{S}:=\sum_{i=1}^{d} w_{i} \cdot \mathbf{1}_{S=\{i\}}+\sum_{|I| \geq 2, S \subseteq I \subseteq \mathbb{D}}(-1)^{|I|-|S|} \beta_{I} \geq 0, \quad \forall S \subseteq \mathbb{D}, S \neq \emptyset .
$$

(II) Conversely, if $\mu_{S} \geq 0$ for any $S \subseteq \mathbb{D}$ with $S \neq \emptyset$, then the DM copula

$$
\tilde{C}\left(u_{1}, \ldots, u_{d}\right)=\left(1-\frac{\mu}{\kappa}\right) C\left(D_{1}\left(u_{1}\right), \ldots, D_{d}\left(u_{d}\right)\right)+\sum_{S \subseteq \mathbb{D}, S \neq \emptyset} \frac{\mu_{S}}{\kappa} C^{(S)}\left(D_{S 1}\left(u_{1}\right), \ldots, D_{S d}\left(u_{d}\right)\right)
$$

satisfies that $b\left(w_{i}, i \in I ; \tilde{C}_{I}\right)=\beta_{I}$ for all $I \subseteq \mathbb{D}$ with $|I| \geq 2$, where $\mu=\sum_{S \neq \emptyset} \mu_{S}, \kappa>(d+1) \mu$, the component copula

$$
C^{(S)}\left(u_{1}, \ldots, u_{d}\right)=\left(\min _{i \in S} u_{i}\right) \cdot \prod_{j \notin S} u_{j}
$$

and distortion functions

$$
D_{i}(x)=\frac{\kappa x-w_{i} D_{i}^{*}(x)}{\kappa-w_{i}} \quad \text { and } \quad D_{S i}(x)= \begin{cases}D_{i}^{*}(x), & i \in S \\ D_{i}(x), & i \notin S\end{cases}
$$


for any $i \in \mathbb{D}$ and $S \subseteq \mathbb{D}, S \neq \emptyset$, in which

$$
D_{i}^{*}(x)= \begin{cases}1-(1-x)^{\frac{\kappa}{w_{i}}}, & w_{i}>0 \\ x, & w_{i}=0\end{cases}
$$

Furthermore, we have

$$
\sup _{\left(u_{1}, \ldots, u_{d}\right) \in[0,1]^{d}}\left|\tilde{C}\left(u_{1}, \ldots, u_{d}\right)-C\left(u_{1}, \ldots, u_{d}\right)\right| \leq \mu(d+1) / \kappa .
$$

Proof. (I) Suppose there is a copula $C$ such that $b\left(w_{i}, i \in I ; C_{I}\right)=\beta_{I}$ for all $I \subseteq \mathbb{D},|I| \geq 2$. Comparing (19) with (20) and applying the definition $b\left(w_{i} ; C_{\{i\}}\right)=w_{i}$, we obtain $\mu_{S}=e_{S}\left(w_{1}, \ldots, w_{d} ; C\right) \geq$ 0 for any $S \subseteq \mathbb{D}, S \neq \emptyset$.

(II) From (20) and the definition of $\mu$, we know that $\kappa \geq \mu \geq \mu_{\{i\}}=w_{i}$ for $i=1, \ldots, d$, which derives that $D_{i}$ and $D_{S i}$ are all distortion functions. And it is easy to verify that Assumption A holds with respect to $\tilde{C}$. Thus according to Theorem 1 , the function $\tilde{C}$ defined in (21) is indeed a copula function.

Let $I \subseteq \mathbb{D}$ with $|I| \geq 2$. As for the TDF of the $I$-marginal copula of $\tilde{C}$, we have

$$
b\left(w_{i}, i \in I ; \tilde{C}_{I}\right)=\lim _{u \downarrow 0}\left(\left(1-\frac{\mu}{\kappa}\right) C_{I}\left(D_{i}\left(w_{i} u\right), i \in I\right)+\sum_{S \in \mathbb{D}, S \neq \emptyset} \frac{\mu_{S}}{\kappa} C_{I}^{(S)}\left(D_{S i}\left(w_{i} u\right), i \in I\right)\right) / u .
$$

For any $i=1, \ldots, d$, we notice that $\lim _{u \downarrow 0} D_{i}(u) / u=D_{i}^{\prime}(0+)=0$, hence

$$
\lim _{u \downarrow 0} C_{I}\left(D_{i}\left(w_{i} u\right), i \in I\right) / u \leq \sum_{i \in I} \lim _{u \downarrow 0} D_{i}\left(w_{i} u\right) / u=0 .
$$

On the other hand, it is easy to verify that $\lim _{u \downarrow 0} D_{i}^{*}(u) / u=\kappa$. Therefore, we can derive

$$
\lim _{u \downarrow 0} C_{I}^{(S)}\left(D_{S i}\left(w_{i} u\right), i \in I\right) / u=\lim _{u \downarrow 0}\left(\min _{i \in I \cap S} D_{i}^{*}\left(w_{i} u\right) \prod_{j \in I \backslash S} D_{i}\left(w_{i} u\right)\right) / u= \begin{cases}\kappa, & I \subseteq S ; \\ 0, & I \backslash S \neq \emptyset .\end{cases}
$$

Applying (25),(26) to (24) and combining with the definition of $\mu_{S}$ in (20), we obtain that for any $I \subseteq \mathbb{D},|I| \geq 2$,

$$
\begin{aligned}
b\left(w_{i}, i \in I ; \tilde{C}_{I}\right) & =\sum_{I \subseteq S \subseteq \mathbb{D}} \frac{\mu_{S}}{\kappa} \cdot \kappa=\sum_{I \subseteq S \subseteq \mathbb{D}} \mu_{S}=\sum_{I \subseteq S \subseteq \mathbb{D}} \sum_{S \subseteq J \subseteq \mathbb{D}}(-1)^{|J|-|S|} \beta_{J} \\
& =\sum_{I \subseteq J \subseteq \mathbb{D}} \beta_{J} \sum_{S: I \subseteq S \subseteq J}(-1)^{|J|-|S|}=\sum_{I \subseteq J \subseteq \mathbb{D}} \beta_{J} \cdot \mathbf{1}_{I=J}=\beta_{I} .
\end{aligned}
$$

And the last inequality in (23) can be obtained from Theorem 1.

Recall that $b\left(1, \ldots, 1 ; C_{I}\right)=\lambda_{C_{I}}$. Thus we can obtain the necessary and sufficient conditions for a group of numbers $\left\{\beta_{I}: I \subseteq \mathbb{D},|I| \geq 2\right\}$ being a marginal TDC family.

Corollary 1. For a group of numbers $\left\{\beta_{I}: I \subseteq \mathbb{D},|I| \geq 2\right\}$, there exists a d-dimensional copula $C$ such that $\lambda_{C_{I}}=\beta_{I}$ for any $I \subseteq \mathbb{D}$ with $|I| \geq 2$, if and only if

$$
\mathbf{1}_{|S|=1}+\sum_{|I| \geq 2, S \subseteq I \subseteq \mathbb{D}}(-1)^{|I|-|S|} \beta_{I} \geq 0, \quad \forall S \subseteq \mathbb{D}, S \neq \emptyset .
$$


Let us see the trivariate case as an example, and details of its proof is moved to the appendix.

Example 2. Let $\beta_{123}, \beta_{12}, \beta_{23}, \beta_{13} \in[0,1]$, there exists a trivariate copula $C$ such that $\lambda_{C_{\{1,2\}}}=\beta_{12}$, $\lambda_{C_{\{2,3\}}}=\beta_{23}, \lambda_{C_{\{1,3\}}}=\beta_{13}$ and $\lambda_{C_{\{1,2,3\}}}=\beta_{123}$ if and only if

$$
\max \left\{0, \beta_{12}+\beta_{23}+\beta_{13}-\min \left\{\beta_{12}, \beta_{23}, \beta_{13}\right\}-1\right\} \leq \beta_{123} \leq \min \left\{\beta_{12}, \beta_{23}, \beta_{13}\right\} .
$$

Theorem 3 shows that DMM enables us to construct copulas with arbitrary marginal TDC family, which provides a theoretical tool for problems about the tail dependence structure. It can be applied in risk management such as Value-at-Risk (VaR) modeling. VaR is the most commonly used risk measure. VaR of a variable $X$ at level $\alpha \in(0,1)$ is given by $\operatorname{VaR}_{\alpha}[X]=$ $\inf \left\{x \in \mathbb{R}: F_{X}(x) \geq \alpha\right\}$, see McNeil et al. (2005) for more introduction about VaR. In the next we will apply the result of Theorem 3 to obtain a tight bound for asymptotic VaR of order statistics. Order statistics is related to many structural products in finance (Hull and White, 2004), and distributional bounds of order statistics can be found in Caraux and Gascuel (1992) and Rychlik (1995). Next we discuss the best possible upper bounds of VaR under the framework of regular variation. We call a random variable $X$ varying regularly at $\infty$ with index $-\delta<0$ if its survival distribution function $\bar{F}_{X}$ satisfies that

$$
\lim _{x \rightarrow \infty} \bar{F}_{X}(t x) / \bar{F}_{X}(x)=t^{-\delta}, \quad \forall t>0 .
$$

For random variables $X_{1}, \ldots, X_{d}$, we denote the order statistics of $X_{1}, \ldots, X_{d}$ as $X_{(1)} \geq \ldots \geq X_{(d)}$.

Theorem 4. Suppose that continuous random variable $X$ varies regularly at $\infty$ with index $-\delta<0$, and $X_{i}$ has identical distribution with $\mu_{i}+\sigma_{i} \cdot X$ for $i=1, \ldots, d$. Let $0<\sigma_{1} \leq \sigma_{2} \leq \ldots \leq \sigma_{d}$, then we have

$$
\underset{\alpha \downarrow 0}{\limsup } \frac{\operatorname{VaR}_{1-\alpha}\left[X_{(k)}\right]}{\operatorname{VaR}_{1-\alpha}[X]} \leq\left(\min _{1 \leq i \leq k} \sum_{j=1}^{d-k+i} \sigma_{j}^{\delta} / i\right)^{1 / \delta}
$$

Moreover, the bound in (29) is tight. And we can use DMM to construct the survival copula of $\left(X_{1}, \ldots, X_{d}\right)$ to reach this bound.

The above theorem can be proved by applying Theorem 3. The detail of the proof will be given in the appendix. Here we only provide the DM copula which reaches the bound.

We denote $i^{*}=\arg \min _{i \in\{1, \ldots, k\}} \sum_{j=1}^{d-k+i} \sigma_{j}^{\delta} / i$ and $\gamma=\sum_{j=1}^{d-k+i^{*}} \sigma_{j}^{\delta} / i^{*}$. Then we define

$$
R_{i}= \begin{cases}{\left[\sum_{j=1}^{i-1} \sigma_{j}^{\delta}, \sum_{j=1}^{i} \sigma_{j}^{\delta}\right)(\bmod \gamma),} & 1 \leq i \leq d-k+i^{*} \\ {\left[0, \sigma_{i}^{\delta}\right),} & d-k+i^{*}<i \leq d\end{cases}
$$

where the notation $R=A(\bmod \gamma), A \subseteq \mathbb{R}$ means that $R=\{x \in[0, \gamma): \exists$ integer $z$ s.t. $x+z \gamma \in A\}$. In the appendix, we will prove if the survival copula of $\left(X_{1}, \ldots, X_{d}\right)$ equals the DM copula in (21) with $w_{i}=\sigma_{i}^{\delta}, i=1, \ldots, d$ and

$$
\mu_{S}=m_{l}\left(\bigcap_{i \in S} R_{i} \bigcap_{j \notin S} R_{j}^{c}\right), \quad \forall S \subseteq \mathbb{D}, S \neq \emptyset,
$$

then the upper bound is obtained, here $m_{l}$ is the Lebesgue measure.

Notice that the bound in (29) is irrelevant to the shift parameters $\mu_{i}$, since it is an asymptotic result. In the following, some examples are given to illustrate the bound. 
Example 3. Let $X$ be a continuous random variable varying regularly at $\infty$ with index $-\delta<0$.

(I) If $\sigma_{1}=\ldots=\sigma_{d}=1$, then for $k=1, \ldots, d$,

$$
\max _{X_{i} \sim \mu_{i}+\sigma_{i} \cdot X} \limsup _{\alpha \downarrow 0} \frac{\operatorname{VaR}_{1-\alpha}\left[X_{(k)}\right]}{\operatorname{VaR}_{1-\alpha}[X]}=\left(\frac{d}{k}\right)^{1 / \delta} .
$$

(II) Let $d=3$ and $\sigma_{1}=4, \sigma_{2}=5, \sigma_{3}=6$, then

$$
\max _{X_{i} \sim \mu_{i}+\sigma_{i} \cdot X} \limsup _{\alpha \downarrow 0} \frac{\operatorname{VaR}_{1-\alpha}\left[X_{(2)}\right]}{\operatorname{VaR}_{1-\alpha}[X]}= \begin{cases}\frac{4+5+6}{2}, & \delta=1 ; \\ \left(\frac{4^{2}+5^{2}+6^{2}}{2}\right)^{1 / 2}, & \delta=2 ; \\ \left(4^{3}+5^{3}\right)^{1 / 3}, & \delta=3 .\end{cases}
$$

\section{Empirical example}

In this section, we will analyze daily log-return data (2000-2012) of S\&P 500 Index and FTSE 100 Index (from Bloomberg database). We will fit the empirical data by DM copula to show that the DM copula has significant advantages against Gaussian copula in the tail parts.

We estimate the marginal distributions empirically, and then use the Maximum Likelihood Estimation to estimate the copula parameters. Firstly, we convert the daily return data $\left(R_{t}^{S P X}, R_{t}^{F T S E}\right)$ to pseudo-samples $\left(U_{t}, V_{t}\right)$ (Chen and Fan, 2006), i.e., the pseudo-samples are defined as

$$
U_{t}=\frac{\operatorname{rank}\left(R_{t}^{S P X}\right)}{n+1}=\frac{1}{n+1} \sum_{k=1}^{n} \mathbf{1}_{R_{k}^{S P X} \leq R_{t}^{S P X}}, \quad V_{t}=\frac{\operatorname{rank}\left(R_{t}^{F T S E}\right)}{n+1}=\frac{1}{n+1} \sum_{k=1}^{n} \mathbf{1}_{R_{k}^{F T S E} \leq R_{t}^{F T S E},}
$$

where $n=3211$ is the length of the data. The empirical copula $C^{e m p}(u, v)$ is calculated by

$$
C^{e m p}(u, v)=\frac{1}{n} \sum_{k=1}^{n} \mathbf{1}_{U_{k} \leq u, V_{k} \leq v}
$$

Next we use $\left(U_{t}, V_{t}\right), t=1, \ldots, 3211$ to estimate parametric copulas by Maximum Likelihood Estimation.

The pseudo-samples $\left(U_{t}, V_{t}\right)$ are shown in Figure 3(a), which likes a scatter plot of Gaussian copula. The fitted Gaussian copula satisfies $\hat{\rho}=0.54$. However, the fitted Gaussian copula $C_{\hat{\rho}}^{N}$ is not able to fit the tail parts. As is well known, the lower and upper TDC of Gaussian copula are both equal to zero. Hence in Figure 3(c), the value $C_{\hat{\rho}}^{N}(x, x) / x$ tends to 0 when $x$ approaches 0 , while the empirical data is obviously correlated in the tail parts. In conclusion, Gaussian copula fits the central part well but doesn't fit the tail parts.

Next we use DMM to modify the tail parts of the fitted Gaussian copula. We choose Gaussian, Clayton and Gumbel copula as the component copulas, and choose $H_{\alpha, \beta}^{f}$ defined in equation (10) as the distortion functions. According to Proposition 3, the DM copula

$$
\begin{aligned}
C_{\vartheta}^{D m}\left(u_{1}, u_{2}\right)= & \left(1-\alpha_{2}-\alpha_{3}\right) C_{\rho}^{N}\left(D\left(u_{1}\right), D\left(u_{2}\right)\right)+\alpha_{2} C_{\theta}^{C l}\left(1-H_{\alpha_{2}, \beta_{2}}^{f}\left(1-u_{1}\right), 1-H_{\alpha_{2}, \beta_{2}}^{f}\left(1-u_{2}\right)\right) \\
& +\alpha_{3} C_{\psi}^{G u}\left(H_{\alpha_{3}, \beta_{3}}^{f}\left(u_{1}\right), H_{\alpha_{3}, \beta_{3}}^{f}\left(u_{2}\right)\right)
\end{aligned}
$$


(a) Pseudo Samples



(c) Lower Tail Behavior



(b) Copula Contours



(d) Upper Tail Behavior

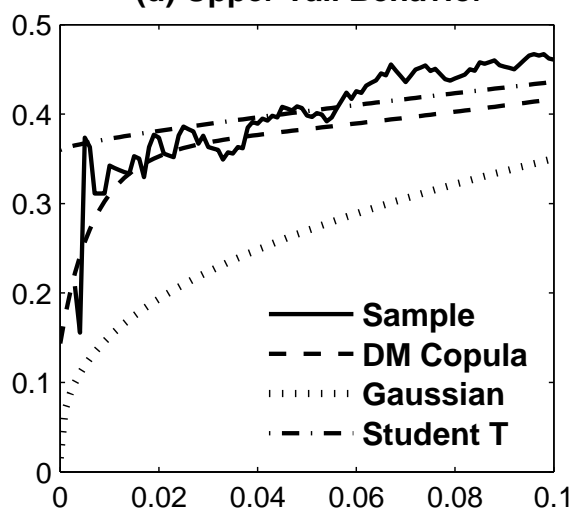

Figure 3: (a) Pseudo-samples of daily log-return data (2000-2012) of S\&P 500 and FTSE 100 Index. (b) Contour lines of empirical copula and the fitted DM copula. Solid lines belong to the empirical copula, and dotted lines belong to the fitted DM copula defined in (32) with parameter $\hat{\vartheta}=$ $(0.54,0.02,0.10,0.56,0.02,5.0,1.1)$. (c) The value $C(u, u) / u$ of four copulas $(u \in[0,0.1])$. The fitted Gaussian copula has correlation parameter 0.54 . The fitted Student T-copula has correlation parameter 0.54 , and its freedom equals 2.7. (d) The value of $\hat{C}(u, u) / u$, where $\hat{C}$ is the survival copula of corresponding copulas.

has identical lower TDF with Clayton copula $C_{\theta}^{C l}$ and identical upper TDF with Gumbel copula $C_{\psi}^{G u}$, where the parameter $\vartheta=\left(\rho, \alpha_{2}, \beta_{2}, \theta, \alpha_{3}, \beta_{3}, \psi\right) \in[0,1] \times\left[0, \frac{1}{2}\right) \times(0, \infty) \times[0, \infty) \times\left[0, \frac{1}{2}\right) \times$ $(0, \infty) \times[1, \infty)$ and $D(x)=\left(x-\alpha_{2}+\alpha_{2} H_{\alpha_{2}, \beta_{2}}^{f}(1-x)-\alpha_{3} H_{\alpha_{3}, \beta_{3}}^{f}(x)\right) /\left(1-\alpha_{2}-\alpha_{3}\right)$. Since we regard the DM copula $C_{\vartheta}^{D m}$ as the tail modified version of the fitted Gaussian copula $C_{\hat{\rho}}^{N}$, we let $\rho=\hat{\rho}=0.54$.

We first set $\alpha_{2}=\alpha_{3}=0.02$, and the other four parameters are estimated by MLE. The estimates are $\hat{\vartheta}=(0.54,0.02,0.10,0.56,0.02,5.0,1.1)$. Since the weight of component Gaussian copula $\alpha_{1}=96 \%$, the fitted DM copula $C_{\hat{\vartheta}}^{D m}(u, v)$ is very close to the fitted Gaussian copula $C_{\hat{\rho}}^{N}(u, v)$. Actually, the mean difference

$$
\int_{0}^{1} \int_{0}^{1}\left|C_{\hat{\rho}}^{N}(u, v)-C_{\hat{\vartheta}}^{D m}(u, v)\right| d u d v=0.0034
$$


Table 1: Results of Maximum Likelihood Estimation.

\begin{tabular}{ccc}
\hline Parameter & Estimate & Std. error \\
\hline$\alpha_{2}$ & 0.0131 & 0.0047 \\
$\beta_{2}$ & 0.1081 & 0.0123 \\
$\theta$ & 0.5594 & 0.0383 \\
$\alpha_{3}$ & 0.0297 & 0.0151 \\
$\beta_{3}$ & 1.5354 & 0.0299 \\
$\psi$ & 1.0856 & 0.0703 \\
\hline
\end{tabular}

which is very small. However, as shown in Figure 3(c) and Figure 3(d), the DM copula $C_{\hat{\vartheta}}^{D m}$ fits the empirical data very well in the tail parts. Comparing with the fitted Student T-copula, DM copula performs well both in the lower and upper tails. Therefore, DM copula shows a great flexibility in the results above, since its lower and upper tails can be calibrated separately.

In the next, we redo the calibration by MLE to estimate the weight parameters $\alpha_{2}, \alpha_{3}$. According to the procedure in Section 2.4, we estimate the parameters $\vartheta=\left(\rho, \alpha_{2}, \beta_{2}, \theta, \alpha_{3}, \beta_{3}, \psi\right)$ through the following steps.

- Step 1: We let $\rho$ equal the correlation parameter of the fitted Gaussian copula, i.e $\rho=\hat{\rho}=$ 0.54 .

- Step 2: We expect $\sup _{(u, v) \in[0,1]^{2}}\left|C_{\hat{\rho}}^{N}(u, v)-C_{\vartheta}^{D m}(u, v)\right| \leq 0.3$. From Theorem 1, we can set $\alpha_{2}, \alpha_{3} \in[0,0.05]$ in advance.

- Then we use MLE to calibrate $\left(\alpha_{2}, \beta_{2}, \theta, \alpha_{3}, \beta_{3}, \psi\right)$ with constraints $\alpha_{2}, \alpha_{3} \in[0,0.05]$.

The MLE estimate $\hat{\vartheta}_{m l}$ of $\vartheta$ is shown in Table 1, the values of $\hat{\vartheta}_{m l}$ have small changes to the previous estimate $\hat{\vartheta}$ discussed in Figure 3, and the fitting effect is also similar.

In order to analyze the fitting effect in different regions, we consider square fit error in region $A \subseteq[0,1]^{2}$ defined as

$$
e_{A}(C)=\left(\frac{1}{m(A)} \iint_{A}\left|C(u, v)-C^{e m p}(u, v)\right|^{2} d u d v\right)^{\frac{1}{2}},
$$

in which $m(A)$ is the Lebesgue measure of set $A, C$ is a fitted copula and $C^{e m p}$ is the empirical copula. The errors of different copulas in different regions are shown in Table 2. From Table 2, we can see that the fitted DM copulas $C_{\hat{\vartheta}}^{D m}$ and $C_{\hat{\vartheta}_{m l}}^{D m}$ perform better in tail parts (in region $[0,0.05]^{2}$ and region $[0.95,1]^{2}$ ) than the fitted Gaussian copula $C_{\hat{\rho}}^{N}$. As for the central region $[0.05,0.95]^{2}$, the fit errors of DM copulas are close to the error of Gaussian copula. Hence these numerical results support our idea of constructing DM copulas to model the tail parts more accurately.

\section{Conclusion}

This paper introduced Distorted Mix Method (DMM) for constructing copula functions by combining the ideas of distortion and convex sum. The constructed DM copula can be very close 
Table 2: Fit error $e_{A}(C)$ of different fitted copula $C$ in different regions.

\begin{tabular}{cccc}
\hline Region & $C_{\hat{\rho}}^{N}$ & $C_{\hat{\vartheta}}^{D m}$ & $C_{\hat{\vartheta}_{m l}^{D m}}^{D m}$ \\
\hline$[0,0.05]^{2}$ & $0.40 \%$ & $0.06 \%$ & $0.07 \%$ \\
{$[0.95,1]^{2}$} & $0.35 \%$ & $0.07 \%$ & $0.06 \%$ \\
{$[0.05,0.95]^{2}$} & $0.63 \%$ & $0.70 \%$ & $0.67 \%$ \\
{$[0,1]^{2}$} & $0.58 \%$ & $0.64 \%$ & $0.62 \%$ \\
\hline
\end{tabular}

to a given copula and has tail dependence as desired. Theoretical properties of the DM copula were discussed by focusing on two tail dependence measures: tail dependence function and tail dependence coefficients of marginal distributions. As an application, a tight bound for asymptotic Value-at-Risk of order statistics was obtained under regular variation framework. Empirical results showed that DM copula fits the empirical data of SPX 500 Index and FTSE 100 Index very well both in central and tail parts.

Acknowledgments. The authors thank a referee for his valuable comments. The research of Kam C. Yuen was supported by a grant from the Research Grants Council of the Hong Kong Special Administrative Region, China (Project No. HKU 7057/13P), and the CAE 2013 research grant from the Society of Actuaries - any opinions, finding, and conclusions or recommendations expressed in this material are those of the authors and do not necessarily reflect the views of the SOA. The research of J. Yang was partly supported by the Key Program of National Natural Science Foundation of China (Grants No. 11131002) and the National Natural Science Foundation of China (Grants No. 11271033).

\section{References}

Caraux, G., Gascuel, O., 1992. Bounds on distribution functions of order statistics for dependent variates. Statistics \& Probability Letters 14, 103-105.

Chen, X., Fan, Y., 2006. Estimation and model selection of semiparametric copula-based multivariate dynamic models under copula misspecification. Journal of Econometrics 135, 125-154.

Denuit, M., Dhaene, J., Goovaerts, M., Kaas, R., 2005. Actuarial Theory for Dependent Risks: Measures, Orders and Models. John Wiley \& Sons Ltd., Chichester.

Donnelly, C., Embrechts, P., 2010. The devil is in the tails: actuarial mathematics and the subprime mortgage crisis. ASTIN Bulletin 40, 1-33.

Durante, F., Foschi, R., Sarkoci, P., 2010. Distorted copulas: constructions and tail dependence. Communications in Statistics: Theory and Methods 39, 2288-2301.

Durante, F., Sempi, C., 2005. Copula and semicopula transforms. International Journal of Mathematics and Mathematical Sciences 2005, 645-655. 
Fischer, M., Köck, C., 2012. Constructing and generalizing given multivariate copulas: a unifying approach. Statistics 46, 1-12.

Genest, C., Rivest, L.P., 2001. On the multivariate probability integral transformation. Statistics \& Probability Letters 53, 391-399.

Hua, L., Joe, H., 2012. Tail comonotonicity: properties, constructions, and asymptotic additivity of risk measures. Insurance: Mathematics and Economics 51, 492-503.

Hull, J., White, A., 2004. Valuation of a CDO and an n-th to default CDS without Monte Carlo. The Journal of Derivatives 12, 8-23.

Joe, H., 1997. Multivariate Models and Dependence Concepts. Chapman \& Hall, Boca Raton.

Joe, H., Li, H., Nikoloulopoulos, A.K., 2010. Tail dependence functions and vine copulas. Journal of Multivariate Analysis 101, 252-270.

Klement, E.P., Mesiar, R., Pap, E., 2005. Transformations of copulas. Kybernetika 41, 425-434.

Klüppelberg, C., Kuhn, G., Peng, L., 2008. Semi-parametric models for the multivariate tail dependence function: the asymptotically dependent case. Scandinavian Journal of Statistics 35, 701-718.

Li, D., 2000. On default correlation: a copula function approach. Journal of Fixed Income 9, 4354.

Liebscher, E., 2008. Construction of asymmetric multivariate copulas. Journal of Multivariate Analysis 99, 2234-2250.

Longin, F., Solnik, B., 2001. Extreme correlation of international equity markets. The Journal of Finance 56, 649-676.

McNeil, A.J., Frey, R., Embrechts, P., 2005. Quantitative Risk Management: Concepts, Techniques and Tools. Princeton University Press, Princeton.

Morillas, P.M., 2005. A method to obtain new copulas from a given one. Metrika 61, 169-184.

Nelsen, R.B., 2006. An Introduction to Copulas, second edition. Springer, New York.

Rychlik, T., 1995. Bounds for order statistics based on dependent variables with given nonidentical distributions. Statistics \& Probability Letters 23, 351-358.

Wang, S., 1996. Premium calculation by transforming the layer premium density. ASTIN Bulletin 26, 71-92.

Yaari, M.E., 1987. The dual theory of choice under risk. Econometrica 55, 95-115. 


\section{A The Proofs}

Proof of Theorem 1. According to Assumption A, it is easy to verify that $\tilde{C}\left(u_{1}, \ldots u_{d}\right)$ has uniform $[0,1]$ margins. To prove $\tilde{C}$ is a $d$-dimensional copula function, we need to check the $d$-increasing property. Since every $D_{i j}$ is increasing, the distorted component copulas $C_{i}\left(D_{i 1}\left(u_{1}\right), \ldots, D_{i d}\left(u_{d}\right)\right)$ are all $d$-increasing. Therefore, $\tilde{C}$ is indeed a copula function.

The maximum difference between $C$ and $C_{i}$ can be estimated directly as

$$
\begin{aligned}
& \sup _{\left(u_{1}, u_{2}, \ldots, u_{d}\right) \in[0,1]^{d}}\left|\tilde{C}\left(u_{1}, u_{2}, \ldots, u_{d}\right)-C_{i}\left(u_{1}, u_{2}, \ldots, u_{d}\right)\right| \\
\leq & \sup _{\left(u_{1}, u_{2}, \ldots, u_{d}\right) \in[0,1]^{d}} \sum_{k=1}^{m} \alpha_{k}\left|C_{k}\left(D_{k 1}\left(u_{1}\right), \ldots, D_{k d}\left(u_{d}\right)\right)-C_{i}\left(u_{1}, u_{2}, \ldots, u_{d}\right)\right| \\
\leq & \sup _{\left(u_{1}, u_{2}, \ldots, u_{d}\right) \in[0,1]^{d}} \alpha_{i}\left|C_{i}\left(D_{i 1}\left(u_{1}\right), \ldots, D_{i d}\left(u_{d}\right)\right)-C_{i}\left(u_{1}, u_{2}, \ldots, u_{d}\right)\right|+\sum_{k \neq i} \alpha_{k} .
\end{aligned}
$$

By using the Lipschitz property of copula function,

$$
\begin{aligned}
& \sup _{\left(u_{1}, u_{2}, \ldots, u_{d}\right) \in[0,1]^{d}} \alpha_{i}\left|C_{i}\left(D_{i 1}\left(u_{1}\right), \ldots, D_{i d}\left(u_{d}\right)\right)-C_{i}\left(u_{1}, \ldots, u_{d}\right)\right| \leq \sum_{j=1}^{d} \sup _{u_{j} \in[0,1]}\left|\alpha_{i} D_{i j}\left(u_{j}\right)-\alpha_{i} u_{j}\right| \\
= & \sum_{j=1}^{d} \sup _{x \in[0,1]}\left|\left(1-\alpha_{i}\right) x-\sum_{k \neq i} \alpha_{k} D_{k j}(x)\right| \leq \sum_{j=1}^{d} \sum_{k \neq i} \alpha_{k} \cdot \sup _{x \in[0,1]}\left|x-D_{k j}(x)\right| \leq \sum_{j=1}^{d} \sum_{k \neq i} \alpha_{k} .
\end{aligned}
$$

Combining all the inequalities above, we have

$$
\sup _{\left(u_{1}, u_{2}, \ldots, u_{d}\right) \in[0,1]^{d}}\left|\tilde{C}\left(u_{1}, \ldots u_{d}\right)-C_{i}\left(u_{1}, \ldots, u_{d}\right)\right| \leq \sum_{j=1}^{d} \sum_{k \neq i} \alpha_{k}+\sum_{k \neq i} \alpha_{k}=\left(1-\alpha_{i}\right)(d+1),
$$

hence the proof of the inequality (2) is completed.

Before we prove the $L^{1}$ inequality in (3), we need to prove that

$$
\int_{0}^{1}\left|x-D_{i j}(x)\right| d x \leq \frac{1}{2}
$$

for any $i$ and $j$. Fixed $i$ and $j$, we denote $y=D_{i j}\left(\frac{1}{2}\right)$. If $y \geq \frac{1}{2}$, then

$$
\begin{aligned}
\int_{0}^{1}\left|x-D_{i j}(x)\right| d x & =\int_{0}^{\frac{1}{2}}\left|x-D_{i j}(x)\right| d x+\int_{\frac{1}{2}}^{y}\left|x-D_{i j}(x)\right| d x+\int_{y}^{1}\left|x-D_{i j}(x)\right| d x \\
& \leq \int_{0}^{\frac{1}{2}}(y-x) d x+\int_{\frac{1}{2}}^{y}(1-x) d x+\int_{y}^{1}(1-y) d x \leq \frac{1}{2}
\end{aligned}
$$


For the case $y<\frac{1}{2}$, we have similar argument to prove (33). By using (33), we have

$$
\begin{aligned}
& \left.\int_{[0,1]^{d}}\left|\tilde{C}\left(u_{1}, u_{2}, \ldots, u_{d}\right)-C_{i}\left(u_{1}, u_{2}, \ldots, u_{d}\right)\right| d u_{1} \ldots d u_{d}\right) \\
\leq & \int_{[0,1]^{d}} \sum_{k=1}^{m} \alpha_{k}\left|C_{k}\left(D_{k 1}\left(u_{1}\right), \ldots, D_{k d}\left(u_{d}\right)\right)-C_{i}\left(u_{1}, u_{2}, \ldots, u_{d}\right)\right| d u_{1} \ldots d u_{d} \\
\leq & \alpha_{i} \int_{[0,1]^{d}}\left|C_{i}\left(D_{i 1}\left(u_{1}\right), \ldots, D_{i d}\left(u_{d}\right)\right)-C_{i}\left(u_{1}, u_{2}, \ldots, u_{d}\right)\right| d u_{1} \ldots d u_{d}+\left(1-\alpha_{i}\right) \\
\leq & \sum_{j=1}^{d} \int_{0}^{1} \alpha_{i}\left|D_{i j}(x)-x\right| d x+\left(1-\alpha_{i}\right)=\sum_{j=1}^{d} \int_{0}^{1}\left|\sum_{k \neq i} \alpha_{k}\left(x-D_{k j}(x)\right)\right| d x+\left(1-\alpha_{i}\right) \\
\leq & d \cdot \sum_{k \neq i} \frac{\alpha_{k}}{2}+\left(1-\alpha_{i}\right)=d\left(1-\alpha_{i}\right) / 2+\left(1-\alpha_{i}\right),
\end{aligned}
$$

so the proof is completed.

Proof of Example 2. According to Corollary 1, we set $S=\{1,2,3\}$ in (27), then we get $\beta_{123} \geq 0$. And we obtain $\beta_{12} \geq \beta_{123}$ by setting $S=\{1,2\}$ in (27). Hence we have $\beta_{23} \geq \beta_{123}$ and $\beta_{13} \geq \beta_{123}$ by the same argument. Therefore, we know that $0 \leq \beta_{123} \leq \min \left\{\beta_{12}, \beta_{23}, \beta_{13}\right\}$.

On the other hand, we set $S=\{1\}$ in (27), then we get $1-\beta_{12}-\beta_{13}+\beta_{123} \geq 0$. Next we set $S=\{2\}$ and $S=\{3\}$ again, we can derive $\beta_{12}+\beta_{23}+\beta_{13}-\min \left\{\beta_{12}, \beta_{23}, \beta_{13}\right\}-1 \leq \beta_{123}$. Combining these results, we obtain the inequalities in (28).

Proof of Theorem 4. Let $i^{*}=\arg \min _{1 \leq i \leq k} \sum_{j=1}^{d-k+i} \sigma_{j}^{\delta} / i$, then we denote $\gamma=\sum_{j=1}^{d-k+i^{*}} \sigma_{j}^{\delta} / i^{*}=$ $\min _{1 \leq i \leq k} \sum_{j=1}^{d-k+i} \sigma_{j}^{\delta} / i$. Denote $F_{i}, F_{(i)}$ as the distribution functions of $X_{i}, X_{(i)}$ respectively. In the next, we divide the proof into several steps.

Step 1. At first, we convert the conclusion into a distributional version. We will prove that

$$
\max _{X_{i} \sim \mu_{i}+\sigma_{i} \cdot X} \limsup _{x \rightarrow \infty} \bar{F}_{(k)}(x) / \bar{F}_{X}(x)=\gamma
$$

implies

$$
\max _{X_{i} \sim \mu_{i}+\sigma_{i} \cdot X} \limsup _{\alpha \downarrow 0} \frac{\operatorname{VaR}_{1-\alpha}\left[X_{(k)}\right]}{\operatorname{VaR}_{1-\alpha}[X]}=\gamma^{1 / \delta} .
$$

Actually, if $\limsup _{x \rightarrow \infty} \bar{F}_{(k)}(x) / \bar{F}_{X}(x) \leq \gamma$, combining with the assumption that $X$ varies regularly, we derive that for any $\varepsilon>0$,

$$
\underset{\alpha \downarrow 0}{\limsup } \frac{\bar{F}_{(k)}\left(\operatorname{VaR}_{1-\alpha}\left[X_{(k)}\right]\right)}{\bar{F}_{X}\left(\operatorname{VaR}_{1-\alpha}\left[X_{(k)}\right]\right)} \leq \gamma \leq \gamma+\varepsilon=\lim _{\alpha \downarrow 0} \frac{\bar{F}_{X}\left(\operatorname{VaR}_{1-\alpha}[X]\right)}{\bar{F}_{X}\left((\gamma+\varepsilon)^{1 / \delta} \operatorname{VaR}_{1-\alpha}[X]\right)}
$$

Then applying the fact $\lim _{\alpha \downarrow 0} \bar{F}_{(k)}\left(\operatorname{VaR}_{1-\alpha}\left[X_{(k)}\right]\right) / \bar{F}_{X}\left(\operatorname{VaR}_{1-\alpha}[X]\right)=1$, from (36) we know that

$$
\underset{\alpha \downarrow 0}{\limsup } \frac{\bar{F}_{X}\left(\operatorname{VaR}_{1-\alpha}\left[X_{(k)}\right]\right)}{\bar{F}_{X}\left((\gamma+\varepsilon)^{1 / \delta} \operatorname{VaR}_{1-\alpha}[X]\right)} \geq 1,
$$


which implies $\lim \sup _{\alpha \downarrow 0}(\gamma+\varepsilon)^{1 / \delta} \operatorname{VaR}_{1-\alpha}[X] / \operatorname{VaR}_{1-\alpha}\left[X_{(k)}\right] \geq 1$. Since $\varepsilon$ is arbitrary, we can conclude that limsup $\operatorname{su}_{\alpha \downarrow} \operatorname{VaR}_{1-\alpha}\left[X_{(k)}\right] / \operatorname{VaR}_{1-\alpha}[X] \leq \gamma^{1 / \delta}$. Furthermore, by the same argument, if a random vector $\left(X_{1}, \ldots, X_{d}\right)$ satisfies $\lim _{x \rightarrow \infty} \bar{F}_{(k)}(x) / \bar{F}_{X}(x)=\gamma$, we can also conclude that $\lim _{\alpha \downarrow 0} \operatorname{VaR}_{1-\alpha}\left[X_{(k)}\right] / \operatorname{VaR}_{1-\alpha}[X]=\gamma^{1 / \delta}$. Hence we have proved that (34) implies (35), so in the following we will prove (34).

Step 2. Denote $C$ as the survival copula of $X_{1}, \ldots, X_{d}$. We will prove

$$
\lim _{x \rightarrow \infty} \bar{F}_{(k)}(x) / \bar{F}_{X}(x)=\sum_{S \subseteq \mathbb{D},|S| \geq k} e_{S}\left(\sigma_{1}^{\delta}, \ldots, \sigma_{d}^{\delta} ; C\right) .
$$

According to the definition of $e_{S}\left(\sigma_{1}^{\delta}, \ldots, \sigma_{d}^{\delta} ; C\right)$ in (17), we have

$$
\begin{aligned}
& \sum_{S \subseteq \mathbb{D},|S| \geq k} e_{S}\left(\sigma_{1}^{\delta}, \ldots, \sigma_{d}^{\delta} ; C\right) \\
= & \sum_{S \subseteq \mathbb{D},|S| \geq k} \lim _{x \rightarrow \infty} \mathbb{P}\left(\bigcap_{i \in S}\left\{\bar{F}_{i}\left(X_{i}\right) \leq \sigma_{i}^{\delta} \bar{F}_{X}(x)\right\} \bigcap_{j \notin S}\left\{\bar{F}_{j}\left(X_{j}\right)>\sigma_{j}^{\delta} \bar{F}_{X}(x)\right\}\right) / \bar{F}_{X}(x) .
\end{aligned}
$$

On the other hand, it is easy to verify that

$$
\lim _{x \rightarrow \infty} \bar{F}_{(k)}(x) / \bar{F}_{X}(x)=\sum_{S \subseteq \mathbb{D},|S| \geq k} \lim _{x \rightarrow \infty} \mathbb{P}\left(\bigcap_{i \in S}\left\{X_{i} \geq x\right\} \bigcap_{j \notin S}\left\{X_{j}<x\right\}\right) / \bar{F}_{X}(x) .
$$

Comparing (38) with (39), we can derive

$$
\begin{aligned}
& \left|\lim _{x \rightarrow \infty} \frac{\bar{F}_{(k)}(x)}{\bar{F}_{X}(x)}-\sum_{S \subseteq \mathbb{D},|S| \geq k} e_{S}\left(\sigma_{1}^{\delta}, \ldots, \sigma_{d}^{\delta} ; C\right)\right| \\
\leq & \sum_{S \subseteq \mathbb{D},|S| \geq k} \lim _{x \rightarrow \infty} \frac{\sum_{i=1}^{d} \mathbb{P}\left(\left\{X_{i} \geq x\right\} \Delta\left\{\bar{F}_{i}\left(X_{i}\right) \leq \sigma_{i}^{\delta} \bar{F}_{X}(x)\right\}\right)}{\bar{F}_{X}(x)} \\
= & \sum_{S \subseteq \mathbb{D},|S| \geq k} \lim _{x \rightarrow \infty} \sum_{i=1}^{d}\left|\frac{\bar{F}_{i}(x)}{\bar{F}_{X}(x)}-\sigma_{i}^{\delta}\right|=0,
\end{aligned}
$$

where the last equality is due to

$$
\lim _{x \rightarrow \infty} \bar{F}_{i}(x) / \bar{F}_{X}(x)=\lim _{x \rightarrow \infty} \bar{F}_{X}\left(\frac{x-\mu_{i}}{\sigma_{i}}\right) / \bar{F}_{X}(x)=\sigma_{i}^{\delta}, \quad i=1, \ldots, d .
$$

Hence the equality in (37) has been proved.

Step 3. We will prove that

$$
\lim _{x \rightarrow \infty} \bar{F}_{(k)}(x) / \bar{F}_{X}(x) \leq \gamma .
$$

Let $C$ be the survival copula of $X_{1}, \ldots, X_{d}$. Recall the supplementary definition $b\left(w_{i} ; C_{\{i\}}\right)=w_{i}$ for $i=1, \ldots, d$. According to the conclusion in (18), we know that

$$
\sigma_{i}^{\delta}=b\left(\sigma_{i}^{\delta} ; C_{\{i\}}\right)=\sum_{S \subseteq \mathbb{D}, i \in S} e_{S}\left(\sigma_{1}^{\delta}, \ldots, \sigma_{d}^{\delta} ; C\right), \quad \forall i=1, \ldots, d .
$$


Therefore, for any $i \in\{1, \ldots, k\}$,

$$
\begin{aligned}
\frac{1}{i} \sum_{j=1}^{d-k+i} \sigma_{j}^{\delta} & =\frac{1}{i} \sum_{j=1}^{d-k+i} \sum_{S \subseteq \mathbb{D}, i \in S} e_{S}\left(\sigma_{1}^{\delta}, \ldots, \sigma_{d}^{\delta} ; C\right) \\
& =\frac{1}{i} \sum_{S \subseteq \mathbb{D}, S \neq \emptyset} e_{S}\left(\sigma_{1}^{\delta}, \ldots, \sigma_{d}^{\delta} ; C\right) \times|S \cap\{1,2, \ldots, d-k+i\}| \\
& =\frac{1}{i} \sum_{S \subseteq \mathbb{D}, S \neq \emptyset} e_{S}\left(\sigma_{1}^{\delta}, \ldots, \sigma_{d}^{\delta} ; C\right)(|S|+d-k+i-|S \cup\{1,2, \ldots, d-k+i\}|) \\
& \geq \frac{1}{i} \sum_{S \subseteq \mathbb{D}, S \neq \emptyset} e_{S}\left(\sigma_{1}^{\delta}, \ldots, \sigma_{d}^{\delta} ; C\right)(|S|+d-k+i-d) \\
& \geq \sum_{S \subseteq \mathbb{D},|S| \geq k} e_{S}\left(\sigma_{1}^{\delta}, \ldots, \sigma_{d}^{\delta} ; C\right) \frac{(|S|+i-k)}{i} \\
& \geq \sum_{S \subseteq \mathbb{D},|S| \geq k} e_{S}\left(\sigma_{1}^{\delta}, \ldots, \sigma_{d}^{\delta} ; C\right)=\lim _{x \rightarrow \infty} \bar{F}_{(k)}(x) / \bar{F}_{d}(x),
\end{aligned}
$$

where the last equality is owing to (37). By the definition $\gamma=\min _{1 \leq i \leq k} \sum_{j=1}^{d-k+i} \sigma_{j}^{\delta} / i$, we obtain (41).

Step 4. We will prove the bound in (41) is tight. Setting $w_{i}=\sigma_{i}^{\delta}$ for $i=1, \ldots, d$ and using the parameters defined in (30),(31), we construct DM copulas $\tilde{C}$ by (21) in Theorem 3. Next we will prove if the survival copula of $X_{1}, \ldots, X_{d}$ is the DM copula $\tilde{C}$, then the upper bound in (41) holds.

Firstly, we prove that

$$
\sigma_{1}^{\delta} \leq \ldots \leq \sigma_{d-k+i^{*}}^{\delta} \leq \gamma
$$

and

$$
\gamma \leq \sigma_{d-k+i^{*}+1}^{\delta} \leq \ldots \leq \sigma_{d}^{\delta} \quad \text { when } i^{*}<k .
$$

Recall the assumption $\sigma_{1} \leq \ldots \leq \sigma_{d}$. For the case $i^{*}<k$, the definition $\gamma=\sum_{j=1}^{d-k+i^{*}} \sigma_{j}^{\delta} / i^{*}=$ $\min _{1 \leq i \leq k} \sum_{j=1}^{d-k+i} \sigma_{j}^{\delta} / i$ implies that

$$
\sum_{j=1}^{d-k+i^{*}+1} \frac{\sigma_{j}^{\delta}}{i^{*}+1} \geq \gamma
$$

this inequality can be simplified as $\gamma \leq \sigma_{d-k+i^{*}+1}^{\delta}$, hence (43) holds. On the other hand, for $i^{*}>1$, by the definition of $\gamma$, we also know

$$
\sum_{j=1}^{d-k+i^{*}-1} \frac{\sigma_{j}^{\delta}}{i^{*}-1} \geq \gamma
$$

which implies $\gamma \geq \sigma_{d-k+i^{*}}^{\delta}$. And if $i^{*}=1$, then $\gamma=\sum_{j=1}^{d-k+1} \sigma_{j}^{\delta} \geq \sigma_{d-k+1}^{\delta}$. Therefore, we have proved (42).

Secondly, we will prove that for any $x \in[0, \gamma), x$ exactly belongs to different $i^{*}$ sets among $\left\{R_{1}, \ldots, R_{d-k+i^{*}}\right\}$. Recall the definitions $\gamma=\sum_{j=1}^{d-k+i^{*}} \sigma_{j}^{\delta} / i^{*}$ and $R_{i}=\left[\sum_{j=1}^{i-1} \sigma_{j}^{\delta}, \sum_{j=1}^{i} \sigma_{j}^{\delta}\right)(\bmod \gamma)$ 
for $i=1, \ldots, d-k+i^{*}$. Thus, for any $x \in[0, \gamma)$, the $i^{*}$ numbers

$$
\left\{x, x+\gamma, x+2 \gamma, \ldots, x+\left(i^{*}-1\right) \gamma\right\} \subseteq\left[0, i^{*} \gamma\right)=\bigcup_{i=1}^{d-k+i^{*}}\left[\sum_{j=1}^{i-1} \sigma_{j}^{\delta}, \sum_{j=1}^{i} \sigma_{j}^{\delta}\right) .
$$

Owing to the result in (42), the length of each interval among

$$
\left\{\left[\sum_{j=1}^{i-1} \sigma_{j}^{\delta}, \sum_{j=1}^{i} \sigma_{j}^{\delta}\right): i=1, \ldots, d-k+i^{*}\right\}
$$

is smaller than $\gamma$. Hence the $i^{*}$ numbers $\left\{x, x+\gamma, x+2 \gamma, \ldots, x+\left(i^{*}-1\right) \gamma\right\}$ belong to different $i^{*}$ intervals among (44). Since $R_{i}=\left[\sum_{j=1}^{i-1} \sigma_{j}^{\delta}, \sum_{j=1}^{i} \sigma_{j}^{\delta}\right)(\bmod \gamma)$ for $i=1, \ldots, d-k+i^{*}$, we conclude that for any $x \in[0, \gamma), x$ exactly belongs to different $i^{*}$ sets among $\left\{R_{1}, \ldots, R_{d-k+i^{*}}\right\}$.

On the other hand, combining the result in (43) with the definition $R_{i}=\left[0, \sigma_{i}^{\delta}\right), i=d-k+i^{*}+$ $1, \ldots, d$, we know that $[0, \gamma) \subseteq R_{i}$ for any $i \in\left\{d-k+i^{*}+1, \ldots, d\right\}$. So for any $x \in[0, \gamma), x$ exactly belongs to different $k$ sets among $\left\{R_{1}, \ldots, R_{d}\right\}$, which implies

$$
[0, \gamma) \subseteq \bigcup_{S \subseteq \mathbb{D},|S|=k}\left(\bigcap_{i \in S} R_{i} \bigcap_{j \notin S} R_{j}^{c}\right) \subseteq \bigcup_{S \subseteq \mathbb{D},|S| \geq k}\left(\bigcap_{i \in S} R_{i} \bigcap_{j \notin S} R_{j}^{c}\right)
$$

Note that $\cap_{i \in S} R_{i} \cap_{j \notin S} R_{j}^{c}$ is pairwise disjoint for $S \subseteq \mathbb{D}, S \neq \emptyset$. Combining with the definition of $\mu_{S}$ in (31), we have

$$
\sum_{S \subseteq \mathbb{D},|S| \geq k} \mu_{S}=\sum_{S \subseteq \mathbb{D},|S| \geq k} m_{l}\left(\bigcap_{i \in S} R_{i} \bigcap_{j \notin S} R_{j}^{c}\right) \geq m_{l}([0, \gamma))=\gamma
$$

As the proof of Theorem 3 part (I), we know that $\mu_{S}=e_{S}\left(\sigma_{1}^{\delta}, \ldots, \sigma_{d}^{\delta} ; \tilde{C}\right)$ for $S \subseteq \mathbb{D}, S \neq \emptyset$ by setting $w_{i}=\sigma_{i}^{\delta}, i=1, \ldots, d$ and comparing the definition in (20) with the expression in (19), Therefore, from (37) we obtain

$$
\lim _{x \rightarrow \infty} \bar{F}_{(k)}(x) / \bar{F}_{d}(x)=\sum_{S \subseteq \mathbb{D},|S| \geq k} e_{S}\left(\sigma_{1}^{\delta}, \ldots, \sigma_{d}^{\delta} ; \tilde{C}\right)=\sum_{S \subseteq \mathbb{D},|S| \geq k} \mu_{S} \geq \gamma
$$

Combining the above results in Step 1 to Step 4, from (41) and (47) we get $\lim _{x \rightarrow \infty} \bar{F}_{(k)}(x) / \bar{F}_{X}(x)=$ $\gamma$. Then according to the proved relationship between (34) and (35), we can get the theorem. 